\title{
Von Biomineralien zu biomimetischen Materialien: Der Weg ist das Ziel
}

\author{
Dirk Volkmer
}

\begin{abstract}
Wie gelingt es lebenden Organismen, aus anorganischen Materialien so komplexe Gebilde wie Stacheln, Schalen oder Muscheln aufzubauen? Von der Aufklärung dieser Mineralisationsprozesse erhofft man sich Anstöße für die Materialwissenschaften.
\end{abstract}

$\mathrm{M}$ it dem Begriff „Biomineralisation” bezeichnen wir im weitesten Sinne den Vorgang der Strukturierung anorganischer Materie durch biologische Formprozesse. Beispiele dafür finden sich bei nahezu allen Lebensformen. Die vergleichsweise harten Biomineralicn übcrnchmen zumcist eine Stütz- oder Schutzfunktion, welche das weiche organische Gewebe vor Deformation durch Druck, Zug-oder Scherkräfte bewahrt.

Einzellige Lebewesen (Protisten) verwenden zum Aufbau von Exoskeletten (Schuppen, Stacheln, Schalen) vorzugsweise Calciumcarbonat $\left(\mathrm{CaCO}_{3}\right)$ oder hydratisiertes Siliciumdioxid ( $\mathrm{SiO}_{2} \times x \mathrm{H}_{2} \mathrm{O}$, mit $x \ll 1$ ). Während niedere Organismen wie die Weichtiere ( $\mathrm{Mol}$ lusken) zum Aufbau kompakter Schalen (Muscheln, Schneckengehäuse) ebenfalls Calciumcarbonat verwenden, findet man bei den Endoskeletten höher entwickelter Wirbeltiere (Vertebrate) Hydroxyapatit, $\mathrm{Ca}_{1}\left(\mathrm{PO}_{4}\right)_{6}(\mathrm{OH})_{2}$, der den Hauptbestandteil der Knochensubstanz ausmacht.

Biomineralien sind stets Verbundwerkstoffe aus einer speziellen organischen Matrix und einer anorganischen Komponente, wobei die organische Matrix einen entscheidenden Einfluß auf die Morphologie der anorganischen Komponente hat. Im Falle kristalliner anorganischer Bestandteile (z. B. Calcit oder Apatit) dient sie der Regulation von Kcimbildung und Kristallwachstum. Darüber hinaus wird das Gefüge der einzelnen Kristalle durch die organische Matrix zusammengehalten (Adhäsion). Die Matrix bildet hier außerdem einen clastischen Schutzüberzug, der den Abbau des anorganischen Formkörpers verhindert.

Auch bei den amorphen Biomineralien, deren anorganischer Anteil aus einem glasartigen,

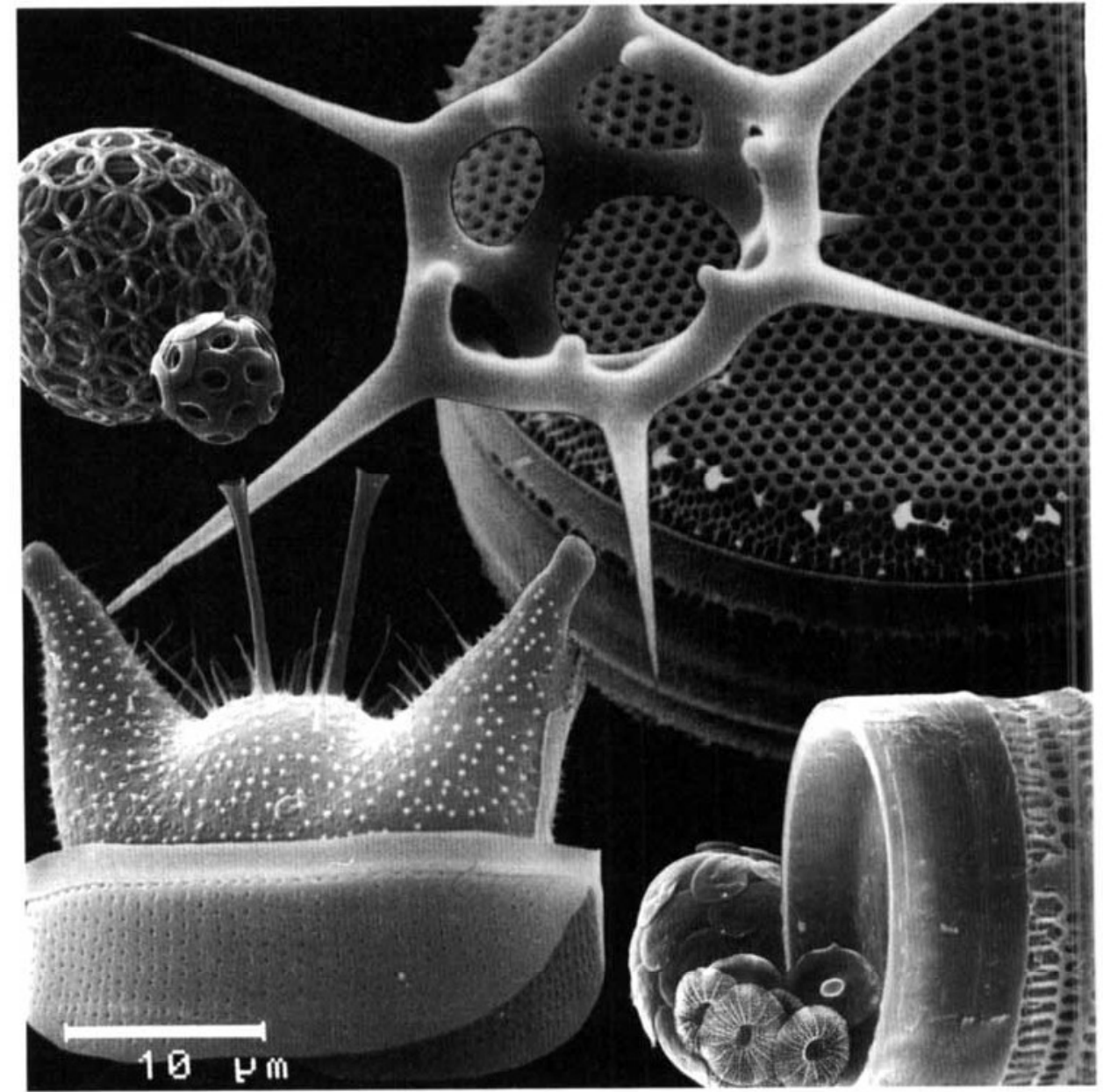

Abb. 1. Viele biologische Organismen nutzen anorganische Materialien zum Aufbau komplex strukturierter Schalen. Die verblüffende Strukturvielfalt erschließt sich dem Beobachter häufig erst bei einem Blick durch das Mikroskop. Hier: REM-Aufnahmen der Schalen einzelliger mariner Mikroorganismen. Obere Reihe (von l. nach r.): Ceratolithus cristatus u. Umbilicosphaera bulburtiana (Coccolithophoride, $\mathrm{CaCO}_{3}$ ), Silicoflagellat (nicht identifizierte Spezies, $\mathrm{SiO}_{2}$ ), Thalassiosira eccentrica (Diatomee, $\mathrm{SiO}_{2}$ ). Untere Reih? (von 1. nach r.): Odontella aurita (Diatomee, $\mathrm{SiO}_{2}$ ), Helicosphaera carteri $\mathrm{u}$. Umbellosphaera tenuis (Coccolithophoride, $\mathrm{CaCO}_{3}$ ), Paralia sulcata (Diatomee, $\mathrm{SiO}_{2}$ ); REM = Rasterelektronenmikroskop. (Fotos: M. Elbrächter, G. Drebes, H. Halliger; BAH) 
2a
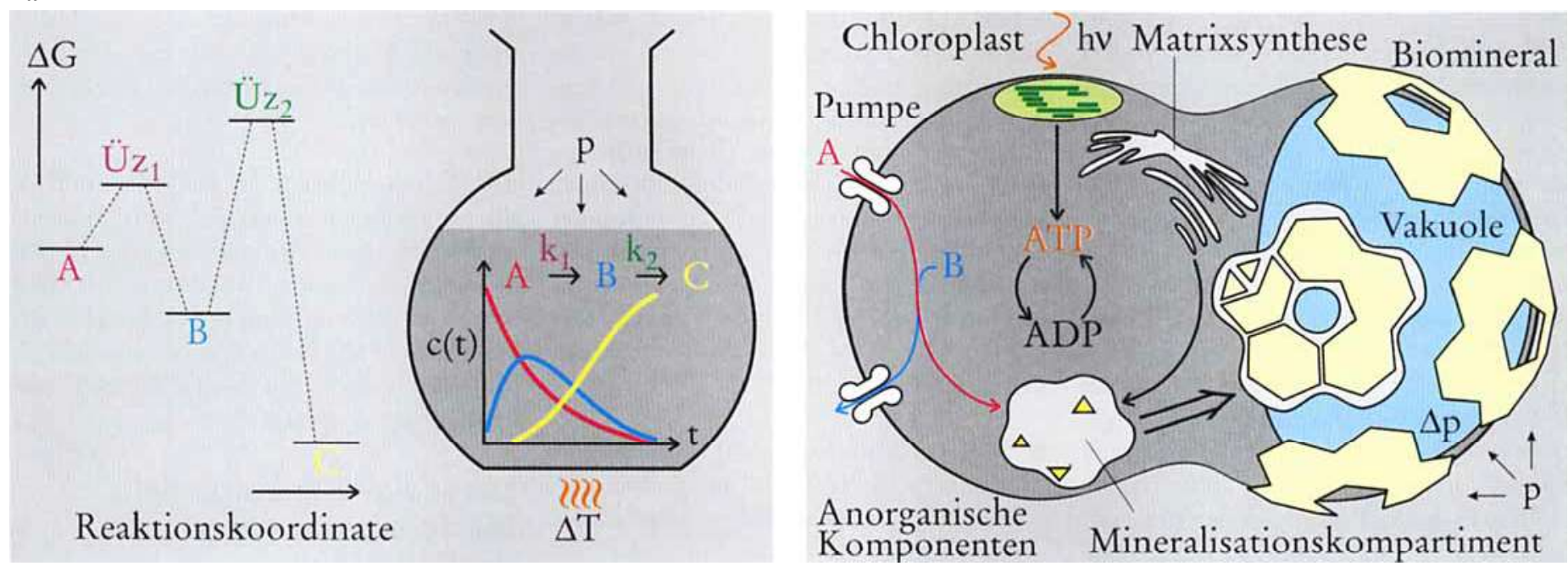

Abb. 2. a) Labormethoden zur Synthese chemischer Produkte beruhen häufig auf der Umsetzung von Stoffen in homogener Lösung. Dabei wird das erwünschte Produkt (hier C) über eine Reihe von Folgereaktionen erhalten. Die Reaktion schreitet stets in der Richtung fort, in der das thermodynamisch stabilere Folgeprodukt gebildet wird (Minimierung der Freien Enthalpie $\Delta G^{\theta}$ ). Die Geschwindigkeit, mit der das thermodynamische Gleichgewicht - und damit der Endzustand der Reaktion - erreicht wird, hängt ausschließlich von der Energiemenge (proportional $\Delta \mathrm{T}$ ) ab, die dem System zugeführt werden muß, damit Aktivierungsbarrieren überwunden werden können, die bei der Stoffumwandlung auftreten (Übergangszustände Üz). Durch Wärmediffusion und Konvektion (Rühren) wird die Energie in dem Reaktor gleichmäßig verteilt. Die Zusammensetzung des Reaktionsgemisches ist in allen Bereichen des Reaktors homogen und die Stoffmenge konstant („stofflich geschlossene Systeme“). b) Im Unterschied zum Laborreaktor wird das Volumen einer Zelle durch biologische Membranen geteilt und gegliedert. Diese Mikrokompartimente haben stoffspezifische Ein- und Ausgänge in Form von membrangebundenen Transportkanälen und Pumpen, mit denen chemische Substanzen innerhalb der Zelle ortsselektiv ausgefiltert oder angereichert werden können („stofflich offene Systeme“). Die erzielten lokalen Konzentrationsungleichgewichte werden durch energieverbrauchende Prozesse aufrecht erhalten, die über (komplizierte) Enzymsysteme an einen energieerzeugenden Prozeß - die Hydrolyse des Adenosintriphosphats (ATP) - gekoppelt sind. Die stoffliche Umwandlung in der Zelle ist daher gekennzeichnet durch eine ungleichmäßige Verteilung von Reaktanden und Energie(-äquivalenten): Chemische Prozesse verlaufen ortsspezifisch, parallel zueinander und in unterschiedlichen Zeitskalen. Das Erkennen derartiger grundlegender Unterschiede ist notwendig, um die komplexe hierarchische Struktur biologischer Materialien zu verstehen; hier schematisch dargestellt als Aufbau einer mineralischen Schale um eine eukaryotische Pflanzenzelle (Erläuterungen s. Text).

Tabelle 1. Biomineralisation als Beispiel zur hierarchischen Organisation der Materie.

\section{Größenebene Stoffhierarchie Wechselwirkung Bcispiel: Schalenbildung}

$\begin{array}{llll}10^{-12} \mathrm{~m} & \text { Atome, Ionen, } & \text { kovalente Bindung, } & \mathrm{Ca}^{2+}, \mathrm{CO}_{3}{ }^{2-} \\ \text { Mikrokosmos } & \text { Moleküle } & \text { Ionenbindung, } & \text { Aminosäuren } \\ & \downarrow & \text { H-Brückenbindungen, } & \downarrow \\ & \text { Supramoleküle } & \text { van der Waals-WW } & \text { Kristallkcim-Protein-Komplex } \\ & \downarrow & & \downarrow \\ & \text { Membran- } & \text { hydrophobe WW, } & \text { Membran-Einkristall- } \\ 10^{-9} \mathrm{~m} & \text { kompartimente, } & \text { kooperative WW, } & \text { Nanokomposite, } \\ \text { Mesokosmos } & \text { Organellen } & \text { z. B. Epitaxie } & \text { Kristalltexturen } \\ & \downarrow & & \downarrow \\ & \text { Zellen } & \text { Adhäsion } & \text { einzelne Bauelemente („Tektone“) } \\ & \downarrow & & \downarrow \\ & \text { Gewcbe, } & \text { Reibung, mechanische } & \text { Bauelement-Verbände } \\ 10^{-6} \mathrm{~m} & \text { Passung, Gravitation } & \text { (,'Tektonik, Architcktur“), } \\ \text { Makrokosmos } & \text { Organe, } & & \text { vollständige Schale }\end{array}$

nichtkristallinen Baumaterial besteht, wird die Gestalt der anorganischen Komponente durch eine organische Matrix geprägt. Es ist eines der ungelösten Rätsel der Natur, wie cine ursprünglich strukturlose Substanz in eine komplexe makroskopische Form gebracht wird, die unserem Empfinden nach wesentliche Merkmale einer "bewußt" ge gliederten Architektur zeigt. Dabei entwickeln bereits einzellige Organismen eine erstaunliche Vielfalt an Strukturen und Formen, deren rätselhafte Schönheit den $\mathrm{Be}$ trachter immer wieder verblüfft (Abbildung 1). Die biologischen Funktionen der häufig anzutreffenden Stacheln, Röhren, Schuppen und Poren sind in den meisten Fällen noch unbekannt; so dürfte z. B. bei der häufig zu beobachtenden Ausbildung langer Stacheln cher die Verminderung der Sinkgeschwindigkeit (wichtig bei marinen photosynthetisicrenden Organismen, die im Oberflächenwasser der Ozeane treiben !) als die Freßabwchr eine Rolle spielen. 
Während sich in der Vergangenheit hauptsächlich Biologen und Mediziner mit Wachstumsprozessen und biogenen Strukturmerkmalen beschäftigten, nimmt das Interesse der sogenannten exakten Wissenschaften (Physik, Mathematik, Chemie) an diesen Phänomenen gegenwärtig stark zu. Die grundlegenden Prinzipien zur Entstehung von Formen und Mustern aufzuklären, ist eines der Hauptzicle cines Forschungszweiges, der zur Zeit die natur- aber auch die sozialwissenschaftlichen Disziplinen durchsetzt und mit dem Arbeitstitel „Prinzipien der Selbstorganisation" überschricben werden kann [1]. Die Bildung von Biomineralien liefert hierzu ein besonders schönes Beispiel, an dem nachvollzogen werden kann, wie sich der Aufbau biologischer Materialien auf verschiedenen Größenebenen vollzicht (vgl. Tabelle 1 und Abbildung 2).

Von besonderer Bedeutung ist dabei der $\mathrm{Be}$ reich der mesoskopischen Systeme, der zwischen der von uns sinnlich wahrnehmbaren Welt und dem Bereich der einfachsten chemischen Einheiten, den Atomen und Molekülen, liegt. In diescm noch weitgehend unerforschten Bereich vollzieht sich der Übergang von der systematisch einfachen und begrenzten zur komplexen und scheinbar uncndlichen Strukturvielfalt.

In der vorliegenden Publikation soll dicser Weg an einem konkreten Beispiel nachvollzogen werden: dem Aufbau hierarchisch strukturierter Schichten und Schalen aus hydratisiertem Siliciumdioxid nach dem Vorbild der Kicselalgen (Diatomeen).

\section{Zelluläre Grundlagen der Biomineralisation}

Über das Gebiet der Biomineralisation als einem neuem Zweig der bioanorganischen Chemie wurde vor einigen Jahren schon an dieser Stelle berichtet [2]. Damals standen die Struktureigenschaften des anorganischen $\mathrm{Ma}$ terials im Mittelpunkt des Interesses.

Biomineralien haben einen komplexen hierarchischen Aufbau, der sich aus dem Ablauf der biologischen Synthese ergibt. Der Mineralisationsprozeß erfolgt in einem offenen $\mathrm{Sy}$ stem $(=$ Zclle mit selektiv permeablen Zellmembranen), fernab vom thermodynamischen Gleichgewicht. Die Zelle befindet sich dabei im ständigen Stoff- und Energieaustausch mit ihrer Umgebung. Bei den cinzclligen Organismen mit einer festen
Schale ist der Mineralisationsprozeß stets an den Zcllcyclus gekoppelt. Dabei werden insbesondere die bei der Zellteilung auftretenden Änderungen des Cytoskelettes und des Zellturgors (Zellinnendruck) geschickt genutzt, um mineralisierte Zellbestandteile zu positionieren oder dauerhaft $\mathrm{zu}$ verformen. Der Aufbau einer mineralisicrten Schale verläuft zeitlich sequentiell und örtlich gerichtet (vektoriell). Dabei lassen sich nach einem groben Schema folgende Stufen unterscheiden (Abbildung 2):

Stufe 1: Die im äußeren Medium gelösten anorganischen Komponenten ( $\mathrm{z}, \mathrm{B} . \mathrm{Ca}^{2+}$, $\mathrm{HCO}_{3}-$-lonen, Kieselsäure) werden über membrangebundene Pumpen in das Zellinnere transportiert. $\mathrm{Um}$ eine spontane Bildung des Biomincrals zu diesem Zeitpunkt zu vermeiden, werden die Mineralbildner zunächst räumlich getrennt angcreichert (z. B. in Speichervesikeln) und/ oder über die Bindung an spezifische Cofaktoren maskiert.

Stufe 2: Parallel zur aktiven Anreicherung der anorganischen Komponenten beginnt die Biosynthese der organischen Mineralisationsmatrix. Dabei handelt es sich um spezielle Makromoleküle wie Polypeptide und Polysaccharide, deren Primärstruktur erst in sehr wenigen Fällen charakterisiert wurde (Tabelle 2).

- Stufe 3: Vereinigung der Mineralisationsmatrix und der anorganischen Komponente( $n)$ in einem speziellen Mineralisationskompartiment - meist eine spezialisierte Vesikel, deren grenzflächenaktive Bestandteile mit dem anorganischen $\mathrm{Mi}$ neral wechselwirken können. Aus der aktuellen Forschungsperspektive wird in der Aufklärung der speziellen statischen und dynamischen Wechselwirkungen zwischen der organischen Mineralisationsmatrix und dem (wachsenden) Mineral der Schlüssel zum Verständnis des Phänomens „Biomineralisation“ geschen. Man nimmt an, daß die Grenzflächen des Minerals und der organischen Matrix kommensurabel sind, d. h. "zueinander passen" - vergleichbar etwa der Beziehung SchlüsselSchloß eines Enzym-Substrat-Komplexes\%.

- Stufe 4: Die primär gebildeten Strukturelemente („Tektone“, z. B. einzclnc Kristallitc) werden zu dreidimensionalen Verbänden ("Assemblagen, Gefüge“) zusammen- geführt, wobei sich eine hierarchische Gliederung des Materials auf mehreren Crößenebenen ergibt, die für die meisten Bio nineralien typisch ist.

Die Biomineralisation ist damit letztendlich als cin spczieller Aspekt der Morphıgenese $\mathrm{zu}$ betrachten, bei der genetisch gespricherte Information (eindimensionaler, molekularer Informationsstring) durch eine konzertierte Abfolge von biochemischen Umwanc lungen in eine makroskopische dreidimersionale Form übertragen wird.

\section{Lebendige Strukturvielfalt: Die Schalenmorphogenese der Diatomeen}

Die bisherigen allgemeinen Aussagen zur Biomineralisation in cinzclligen Orga vismen gehen nicht auf das verwendete Bauntaterial ein. Es ist jedoch leicht verständlich, $d a ß$ sich die molekularen Grundlagen zum tsufbau von Schalen aus kristallinem Material (im Bereich der Mikroorganismen ist dies vorwiegend $\mathrm{CaCO}_{3}$ in den Kristallmodifikitionen Calcit, Aragonit und Vaterit) im Detiil von denjenigen unterscheiden, bei dene s eine amorphe Substanz (hydratisiertes Silic umdioxid) in eine entsprechende Form grbracht wird (vgl. Tabelle 2). Die Situation lä 3t sich grob vereinfachend vergleichen mit $d \geq n$ unterschiedlichen Strategien beim Hausbau: Beim Fachwcrkbau wird die Hausgrurdform mit einem Holzrahmen (organische Matrix) vorgegeben und die Freiräume anschl $\mathrm{c}$ Bend mit einzelnen Backsteinen ( $\mathrm{CaCO}_{3}$-Kristalle) ausgefacht; bei der Spannbauweise vrerden durch Ausfüllen von Negativgußforms n (organische Matrix) mit dem zunächst flicßbcweglichen Beton (amorphes hydratisiertes $\mathrm{SiO}_{2}$ ) großflächige Bauteile vorgefertist und anschließend paßgenau aneinandergefü żt.

* Da hier jedoch gleichzeitig ionische, intramolekulare und intcrmolekulare (ster ochemische) Wechselwirkungen über einen ıtusgedehnten räumlichen Bereich berücks chtigt werden müssen, ist die vollständige Mo tellierung der auftretenden Grenzflächenphänomene mit den zur Zeit üblichen Met ıoden (Kraftfeldmethoden, Moleküldynamik simulationen) sowohl nach theoretischen (das Fehlen geeigneter Kraftfelder) als auct nach praktischen Gesichtspunkten (Compı terrechenleistung) nur eingeschränkt möglic . . 
Tabelle 2. Bestandteile von Biomineralien: eine Auswahl.

Anorganische Komponente Organische Komponente Organismus / Funktion

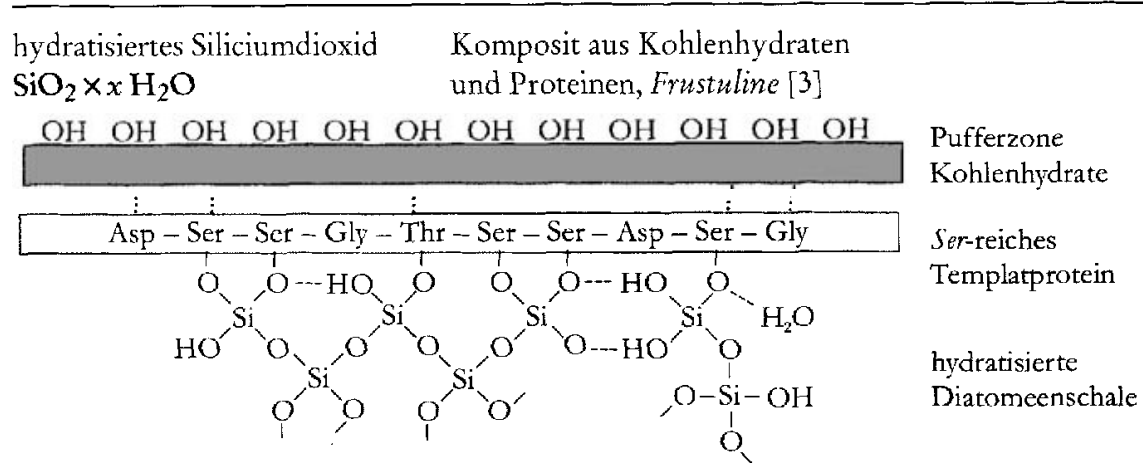

Modell der Diatomeenschale nach Hecky et al. [4]

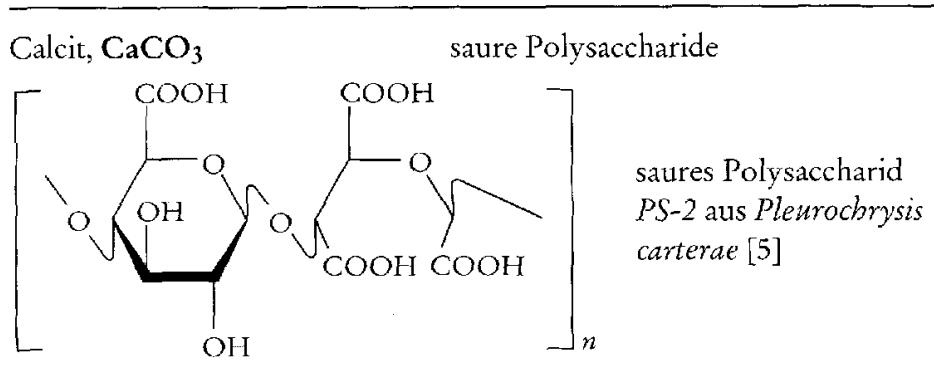

Aragonit, $\mathrm{CaCO}_{3}$

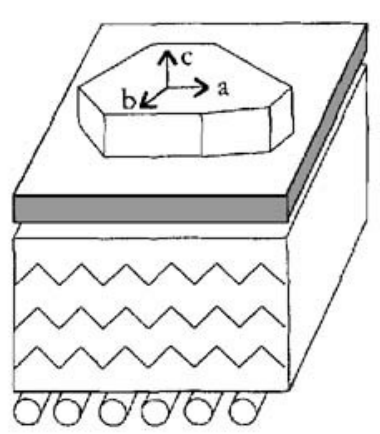

Mikrolaminat aus Kohlenhydraten und Proteinen

Aragonit-Einkristall

wasserlösliche, $A s p$-reiche

Glykoproteine

Polypeptide mit einer dem

Seidenfibroin ähnlichen

Zusammensetzung

(Poly-Ala- und Poly-Gly-Domänen)

B-Chitinfibrillen
Diatomeen / Katalyse der Kondensation der Kieselsäure (?) Adhäsion der Schalenbauteile
Coccolithophoriden / $\mathrm{Ca}^{2+}$-Anreicherung, Förderung der Keimbildung (?)

\section{Hydroxyapatit, $\mathrm{Ca}_{10}\left(\mathrm{PO}_{4}\right)_{6}(\mathrm{OH})_{2}$ \\ Mikrolaminat aus Kollagen-Fibrillen und regulatorischen Proteinen}<smiles></smiles>

Perlmuttschicht der Molluskenschalc /

Templatmatrix zum Kristallaufwuchs
Aufbau einer

Perlmuttschicht

nach Weiner et al. [6]<smiles>CNC(=O)[C@H](CC(=O)[O-])NC(=O)C(C)(C)C(=O)NC</smiles><smiles>[O]</smiles><smiles></smiles>

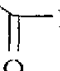

Poly-(Asp-Ser*-Ser*)-Strukturmotiv der Phosphophorine in der Dentinmatrix nach Veis et al. [7]

Knochen, Zähne /

Templatmatrix zum Kristallaufwuchs 
In der Natur finden sich zahlreiche Organismen, die Schalen aus hydratisiertem Siliciumdioxid bilden [8]. Dabei haben insbesondere die mikroskopischen Kiesclalgen (Diatomeen) Mechanismen entwickelt, den Baustoff - die Kieselsäure - in extrem vielfältiger Weise zu formen und zu prägen (vgl. Infokasten auf S. 12/13). Wegen der wichtigen technischen Anwendungen, bei denen poröse Siliciumdioxidmaterialien eine Rolle spielen, etwa als Basis von Trägerkatalysatoren, Adsorbentien und Filtermaterialien [9], ist das wirtschatiliche Interesse an Verfahren zur gezielten Kontrolle der Materialporositä und -morphologie enorm groß. Dic Morphogenese der Diatomeenschale ist dabei aus materialwissenschaftlicher Perspektive eine Leistung, für die es auf seiten der Synthesechemie gegenwärtig keine Entsprechung gibt (Tabclle 3).

Wie gelingt es den einzelligen Diatomeen, ein anorganisches Material wie das $\mathrm{SiO}_{2}$ in die beobachteten, eindrucksvollen Formen zu bringen? Zur Beantwortung dieser Frage müssen wir zunächst die biologischen Vorgänge beschrciben, die sich bei der Morphogenese der Diatomeenschale ereignen. Dabei muß nach der Formbildung, die die äußere Gestalt der Diatomeenzelle bedingt und der Entstehung der charakteristischen Muster unterschieden werden. Die folgende Darstellung schliefst sich ciner cytologischen Sichtweise an, deren Argumente sich in erster Linie auf die sorgfältige Interpretation lichtund elektronenmikroskopischer Aufnahmen der Diatomeenzelle stützen [10].

\section{Die Zellteilung bei den Diatomeen}

Die Bildung der Diatomeenschale ist an den Cyclus der vegetativen Zelltcilung gebunden und crfolgt darin stets nach der Mitose (Abbildung 3). Im Endergebnis entstehen zwei unterschiedlich große Tochterzellen, von denen nur cine die ursprüngliche Größe der Mutterzelle bewahrt. Die Diatomeen teilen sich somit im Laufe der vegetativen Vermehrung allmählich bis zum Erreichen ihrer artspezifischen Minimalgröße. Dabci verändern sich Geometrie, Struktur und Inhalt der Zellen, wodurch die Identifizierung der Zellen erschwert sein kann (Abbildung 4). Der Größenschwund wird durch besondere Reproduktionscyclen wicder ausgeglichen (scxuelle Fortpflanzung, Bildung von Auxosporen), worauf hier aber nur am Rande hingewiesen werden soll (für nähere Informationen siche z. B. [11]).

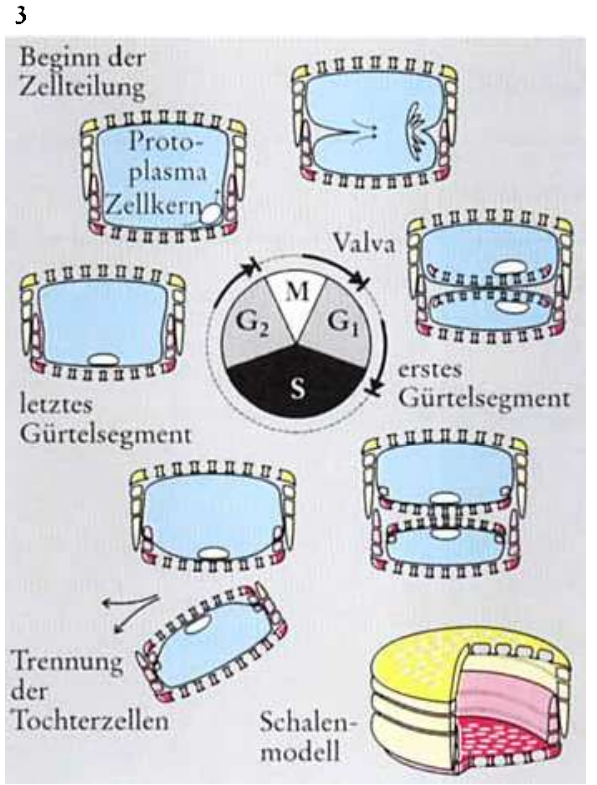

Da sich die neuen Halbschalen der Tochterzellen innerhalb des starren Kicsclskclettes der Mutterzellc bilden, ist cine Übertragung der äußeren Form möglich: Die Schale der Mutterzelle dient bildlich ausgedrückt als Gußform (cngl. mould), in welche das Baumaterial Kiesclsäure hincingepreßt wird. Die Synthese der neuen Halbschalen geschieht in cinem abgegrenzten Zcllkompartiment, der silica deposition vesicle (SDV). Diese sperielle Vesikel wird kurze Zeit nach der Mitose gebildet und wächst als flacher Membransack cntlang der Innenseitc der bei der Zellteilung neu entstandenen Plasmamembran(en) (Abbildung 6). Die SDV entspringt vermutlich dem endoplasmatischen Reticulum der Zelle, der Ursprung liegt zunächst am Mittelpunkt

Tabelle 3. Technische und biologische Synthese strukturierter $\mathrm{SiO}_{2}$-Matrices im Verifleich.

Zeolithsynthese

Tage

Reaktionszeit

Konz. des anorg. Precursors

pH-Wert

Temperatur

Druck

Strukturbeschreibung
Abb. 3. Schema zur vegetativen Zellteilung bei den Diatomeen (nach [10]). D e Zellteilung wird durch ein Anschwelen des Protoplasten eingeleitet. Der Kern bewegt sich in dem dargestellten Beispiel von seiner ursprünglichen Position im Zentrum der Hypotheka zum Überlappungsbere ich der Gürtel. Unmittelbar nach der Kernteilung (Mitose, $M$-Phase) erfolgt die Zellteilung, dabei löst sich der Protoplast in Bertich der Gürtelüberlappung von dem Kieselikelett; gleichzeitig beginnt die Ausbildung neuer Zellwände. Die Bildung der Valven (,Schalendeckel“) und der Gürtelsegmer te geschieht in zeitlich getrennten Schritten. Die Valven der Tochterzellen werden intrazellulär in einer spezialisierten Vesikel (silica deposition vesicle) geformt. Der Auft au der Gürtel zu den Tochterzellen kann s.ch dagegen über die gesamte Interphase erstrecken. Die ursprüngliche Mutierzelle enthält also zunächst die Protoplasten der Tochterzellen mit unvollständig ausgebildeter Kieselschale. Jede Tochterzelle erbt eine Halbschale (die größere Epither a, hell abgesetzt) während die kleineren IJnterschalen (Hypothecen, dunkel) neu gəbildet werden. (Die Interphase wird traditionell untergliedert in die Phasen der DNS- Replikation (S-Phase) und verschiedene Wachstumsphasen ( $G_{1}-$ u. $G_{2}$-Phasen), vgl. allg. einführende Lehrbücher der Zellbiok gie.) 

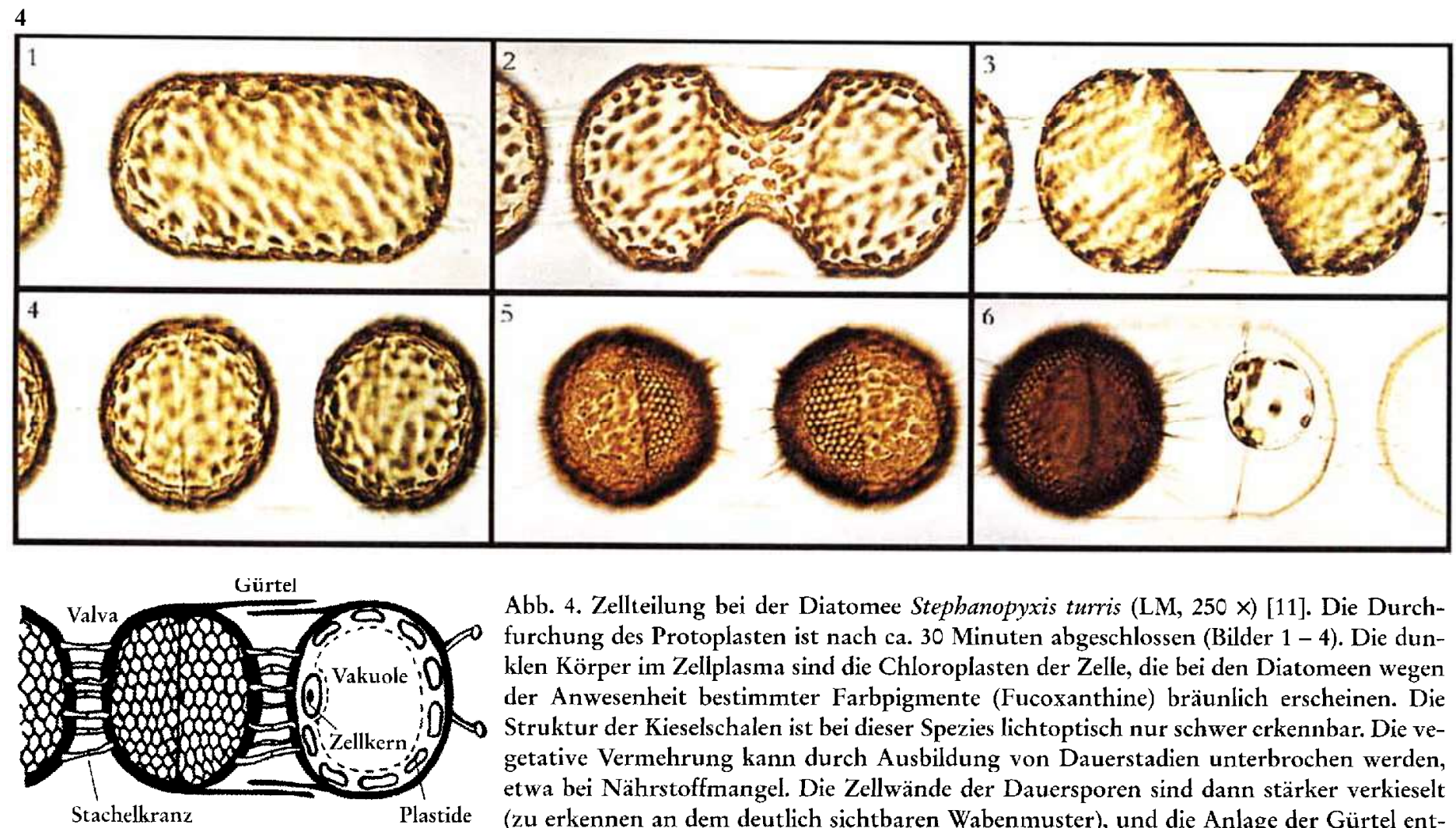

Abb. 4. Zellteilung bei der Diatomee Stephanopyxis turris (LM, $250 \times$ ) [11]. Die Durchfurchung des Protoplasten ist nach ca. 30 Minuten abgeschlossen (Bilder 1 - 4). Die dunklen Körper im Zellplasma sind die Chloroplasten der Zelle, die bei den Diatomeen wegen der Anwesenheit bestimmter Farbpigmente (Fucoxanthine) bräunlich erscheinen. Die Struktur der Kieselschalen ist bei dieser Spezies lichtoptisch nur schwer erkennbar. Die vegetative Vermehrung kann durch Ausbildung von Dauerstadien unterbrochen werden, etwa bei Nährstoffmangel. Die Zellwände der Dauersporen sind dann stärker verkieselt (zu erkennen an dem deutlich sichtbaren Wabenmuster), und die Anlage der Gürtel entfällt (Bilder 5 und 6). (Fotos: G. Drebes, BAH)

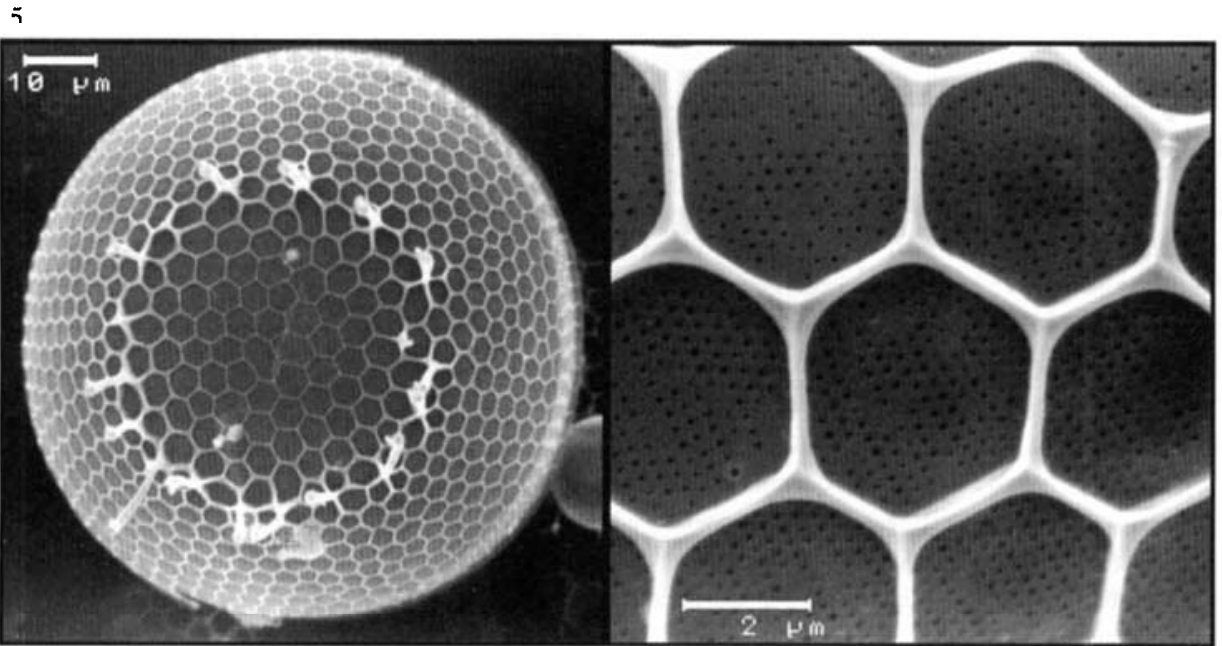

Abb. 6. Modell zum Siliciummetabolismus bei den Diatomeen. Die Bildung der Diatomeenschale erfolgt schrittweise durch Kondensation der im äußeren Nährmedium vorhandenen Kieselsäure $\left(\mathrm{Si}(\mathrm{OH})_{4}\right)$. Die Kieselsäure wird zunächst in monomerer Form über einen aktiven Transporter aufgenommen, der in der äußeren Zellmembran lokalisiert ist [12].

Um die unkontrollierte Polymerisation der Kieselsäure bei der Anreicherung im Zellplasma zu verhindern, erfolgt zunächst eine Maskierung, d. h. chemische Bindung an einen Cofaktor $\left(\mathrm{Si}(\mathrm{OH})_{4} \subset \mathrm{Cof}\right)$, dessen chemische Natur noch unbekannt ist. Die vorübergehend maskierte Kieselsäure wird vermutlich im Golgi-Apparat der Zelle ge-

Abb. 5. Kieselschale der Diatomee Stephanopyxis turris (REM). Gesamtaußenansicht der domförmigen Valva im Normalstadium (links) und Detailansicht der hexagonalen Wabenstruktur (rechts) mit darunter liegender mikroporöser Membran. (Fotos: H. Halliger, BAH)

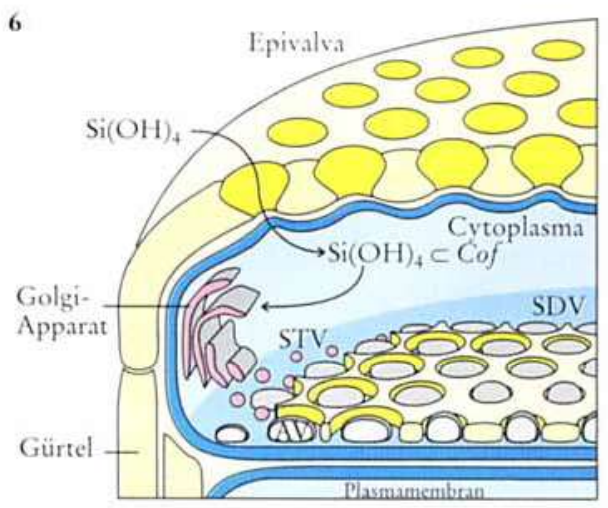
speichert.

Aus dem Kieselsäuredepot des Golgi-Apparates werden bei der Schalenbildung kleinere Transportvesikeln (silica transport vesicles, STV) abgesondert, die mit dem eigentlichen Mineralisationsorganell der Zelle, der silica deposition vesicle (SDV), verschmelzen. In der SDV findet die Kondensation der Kieselsäure statt; Form und Muster der Schale werden durch die Zellmatrix geprägt. (Zeichnung nach Angaben in [13]) 
der späteren I Hypovalva (Bodenfläche der Zellwand), von der sich die Vesikel rasch radiär ausbreitet. Dabei windet sie sich um eine Vielzahl von kleineren Membranausstülpungen, die bei der vorhergehenden Durchfurchung des Zellplasmas entlang der Zellmembran angelegt wurden. Elektronenmikroskopische Studien legen nahe, daß cs sich dabci un kleine Vesikel (areolae vesicles, AV) handelt, deren Anordnung sich häufig als eine einzelne Schicht dicht gepackter Bläschen darstellt. Die dichteste Anordnung von glcichförmigen Kugeln in ciner Ebene wird mit einer hochsymmetrischen hexagonal dichten Packung erreicht; dic sich ausbreitende SDV erhält einen Negativabdruck des Bläschenmusters (engl. pattern), was die sehr häufig zu beobachtende hexagonalc Kammerstruktur (Bienenwabenmuster) der Diatomeevalven zwanglos erklärt (Abbildung 5). Insgesamt wird die Schalenmorphogenese so gesehon, daß das Siliciumdioxid als passives Füllmaterial in eine vorgefertigte Form (die SDV) abgelagert wird (mould-prepatternHypothese). Form und Muster der Diatomeenschale werden über zeitlich synchronisierte Veränderungen des Cytoskelettes vorherbestimmt.

Die kurze Darstellung über die Biomincralisation der Kieselsäure in den Diatomeen wäre an dieser Stelle eigentlich schon beendet. Der vorhergehende Abschnitt macht deutlich: Die Bildung der strulkturicrten Kicselschalc ist cin sehr komplizierter Prozeß, dessen molekulare Grundlagen weitgehend unbekannt sind. Die mould-prepattern-Hypothese der Schalenmorphogenese ist eine Beschreibung der Vorgänge, eine Erklärung liefert sie jedoch nicht. Die Gestalt der Diatomeenschale wird im Zellplasma durch das Wirken unbekannter Kräfte präformiert, die - unter „bewußter" Steuerung vom Zellkern aus - über das Cytoskelctt der Zclle auf dic sich bildende Kieselschale übertragen werden. Das Geheimnis der vielfältigen Muster und Formen wäre dann im Genom der Diatomeenzelle festgeschrieben, und es wäre die Aufgabe zukünftiger molekulargenetischer Studien, diese verschlüsselte Botschaft zu entziffern.

Es gibt berechtigte ' $Z$ weifel, daß die Kenntnis der genetischen Information allein ausreichte, um den Vorgang der Formbildung zu verstehen. Die Einwände sind sowohl theoretischer als auch praktischer Natur. Eine Energiebilanz der Diatomeenzelle zeigt, daß lediglich $2 \%$ der Energie des Gesamtstoffwechsels für dic Bildung ciner ausgereiften Schale benötigt werden - eine Größenordnung weniger als für die Bildung eines vergleichbaren Cellulosegerüstes notwendig ist. Die Ausbildung und Strukturierung eincr vollständigen Schale findet in manchen Fällen auch unabhängig von der Zellteilung statt. In den meisten Diatomeen wird zwar die äußcre Schalcnform an dic Tochtergcnerationen weitergegeben, die Mikrostrukturierung (z. B. die Verteilung und Häufigkeit der Mikroporen) unterlicgt aber individuellen Schwankungen [14]. Es liegt der Verdacht nahe, daß an der Schalenbildung weit weniger komplexe Steuerungsmechanismen betciligt sind, als wir dies zunächst vermuteten.

\section{Lernen von der Natur: der Weg zu biologisch inspirierten Materialien}

Wir können dic Frage nach dem Ursprung der Gestalt auch von einer anderen extremen Position stellen: Gibt es grundlegende (und vermutlich einfache) stoffliche Eigenschaften im „System Diatomeenzelle", die - bei geeigneter Kombination - zu einer spontanen Strukturbildung führen? Zur Beantwortung dieser Frage müssen wir uns kurz den Eigenschaf ten des Baumaterials, dem $\mathrm{SiO}_{2}$, zuwenden.

\section{Die Kondensation der Kieselsäure}

Über die Chemie des Siliciumdioxids in wäßrigen Systemen gibt es umfangreiche Monographien [15], es sollen hier lcdiglich cinige morphologische Aspekte erwähnt werden. Bei neutralem pH-Wert löst sich $\mathrm{SiO}_{2}$ in reinem Wasser bis zu einer Sättigungskonzentration von $2 \mathrm{mM}$ in monomerer Form (Kieselsäure, $\left.\mathrm{Si}(\mathrm{OH})_{4}\right)$. Bei höheren Konzentrationen kommt es zu Kondensationsreaktionen, in deren Verlauf kolloidale Partikeln aus hydratisiertem Siliciumdioxid gebildet werden (Solbildung). Die Aggregation der kolloidalen Partikeln führt zu dreidimensionalen Netzwerken, deren Mikrostrukturierung sich aus der Größc der Kolloidpartikeln und dem Vernetzungsgrad ergibt (Abbildung 7). So führt die Kondensation von nanometcrgroßen, globulären Partikeln zu Kolloid gelen mit ausgeprägter Feinstruktur („Perlenkettc-Struktur"). Dagegen bilden sich Polymergele aus der Quervernetzung linearer und verzweigter eindimensionaler Polymerketten (,Spinnennetz-Struktur“).

Der innere Aufbau der Kieselgele war den Naturforschern vor mehr als 100 Jahren weit

\section{Kieselalgen}

Kieselalgen [36] (Diatomeen, von griech diatomos = zerschnitten, geteilt) sind ein zellige cukaryotische Organismen, die zu meist vereinzelt leben, manchmal aber auch Kolonien bilden. Ihre Zellgröße schwank zwischen Bakteriengröße $(<2 \mu \mathrm{m})$ und Rie senformen mit Durchmessern von mehr al $2 \mathrm{~mm}$. Das auffälligste Merkmal ist die ver kieselte Zellwand (Frustel), die aus zwei sict überlappenden Halbschalen (Theken) be steht, vergleichbar mit ciner Petrischale be stehend aus dem kleineren Schalenboden (= Hypotheka) und dem überlappenden Scha lendeckel (= Eputheka). Dic Klassifizicrun beruht im wesentlichen auf strukturelle Details der Schalenmorphologic, daher exi stiert eine umfangreiche Terminologic fü die silikatischen Komponenten der Zell wand. Nach dem zur Zeit üblichen taxono mischen System bilden die Kieselalgen in Pflanzenreich unter der Abteilung Hetero kontophyta eine eigene Klasse (Bacillario phyceac $=$ Diatomeen $)$. Man unterscheide zwei Ordnungen, die der centrischen Dia tomeen (Centrales) und der pennaten Dia tomeen (Pennales).

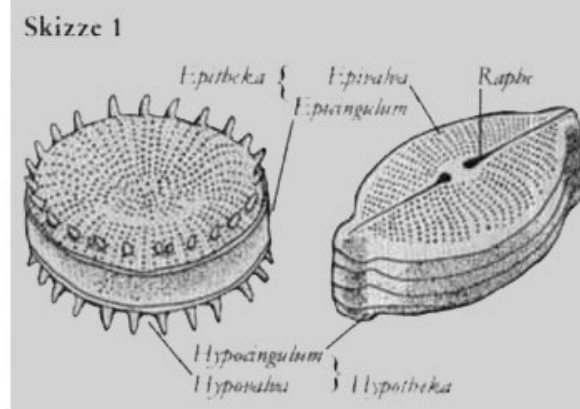

Schematischer Bauplan einer centrischen (links) und einer pennaten (rechts) Diatomee.

Dic beiden Ordnungen umfassen zusam men etwa 250 Gattungen mit weit über 10000 Arten. Die Kieselalgen bilden damit die umfangreichste Klasse der einzelligen Lebewesen (Protisten). Mit ihrer Fähigkeit zur Photosynthese gehören sie zu den wichtigsten Primärproduzenten von orga nischer Substanz und bilden somit die Nah rungsgrundlage für das Leben in Gewäs sern. Kieselalgen kommen in allen Klimazo nen der Erde im Meer-, Brack- und Süßwasser vor; insbesondere besteht das Phytoplankton der lichtdurchfluteten ober flächennahen Zonen der Weltmeere zu ei 
nem großen Teil aus Kieselalgen. Massenvermehrungen von Diatomeen sind leicht an der bräunlichen Färbung der Gewässer erkennbar. Die Farbwirkung wird durch das Pigment Fucoxanthin hervorgerufen, welches die Chloroplastenfarbstoffe Chlorophyll $a$ und $c$ in der Zelle überdeckt. Wichtigste Reservestoffe sind Chrysolaminaran, ein B-1,3-Glucan (Skizze 2), welches in Vakuolen vorkommt, sowie Lipidtröpfchen, die im Plasma verteilt vorliegen.

Kieselalgen sind nach entwicklungsgeschichtlichen Maßstäben eine verhältnismäßig junge Klasse von Organismen. Die ersten Spuren finden sich in der frühen Jurazeit, vor 190 - 180 Millionen Jahren. Es waren zunächst centrische Diatomeen, die sich in der frühen Kreidezeit (vor 140 Millionen Jahren) weit verbreiteten. Erst in der späten Kreidezeit, vor 70 Millionen Jahren, erschienen die pennaten Diatomeen, die sich dann im Eozän stark entwickelten.

Die fossilen Kieselschalen der Diatomeen

Skizze 2

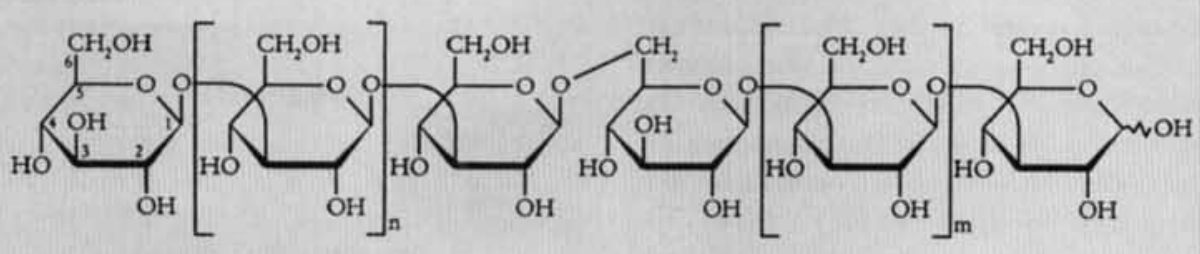

Chrysolaminaran, ein $(1 \rightarrow 3)-\beta$-D-verknüpftes Glucan

weisen eine große Haltbarkeit auf. So entstanden allmählich sedimentäre Ablagerungen von erheblicher Schichtdicke. Besonders reines, von anderen Beimengungen freies Schalenmaterial, hat sich während des Tertiärs (60 Mio) und Quartärs (2 Mio) in flußarmen Binnenseen und flachen Meeren abgesetzt. Diese Kieselerde (Kieselgur, Diatomit) bildet an manchen Stellen Schichten von mehr als $100 \mathrm{~m}$ Dicke, die wirtschaftlich nutzbar sind und in größerem Umfang in Gebieten der ehemaligen Sowjetunion, Nordamerika (Kalifornien), Nordafrika und Großbritannien abgebaut werden. Die Diatomite mit sandiger oder schieferartiger
Struktur werden vorwiegend als Filtermasse, Füllmittel und Isoliermaterial verwendet. Die spektakulärste Verwendung fand das Material durch Alfred Nobel, der es 1876 für die Herstellung des ersten Dynamits benutzte.

Mit Hilfe der Diatomeenanalyse der Sedimente lassen sich wertvolle Schlüsse über den historischen Ablauf von biologischen, vegetationsgeschichtlichen, geologischen und klimatischen Prozessen ziehen. Sie ergänzt die Pollenanalyse und ${ }^{14} \mathrm{C}-\mathrm{Me}$ thodik, um marine und limnische Ablagerungen zeitlich genau datieren zu können.
A

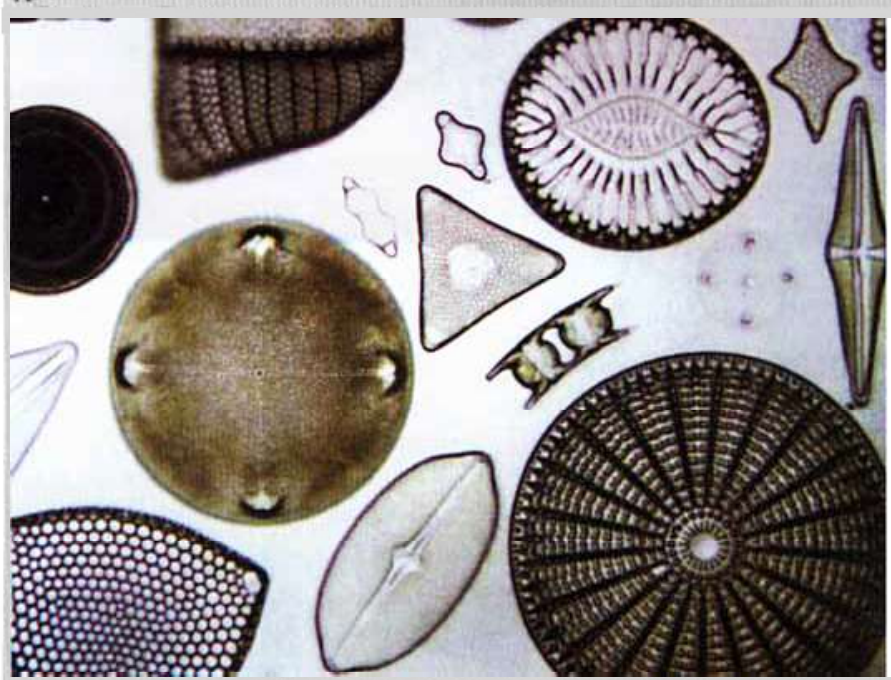

Abb. A. Diatomeen sind die bedeutendsten Biomasse- und Sauerstoffproduzenten im Süßwasser und im Meer. Ihre komplexen, artspezifischen Zellwandstrukturen aus Siliciumdioxid sind von außergewöhnlicher Schönheit. Die mikroskopische Untersuchung von Diatomeen ist eine große Herausforderung, denn oft lassen sich die feinen Strukturen nur mit den besten $\mathrm{Ob}$ jektiven auflösen. $\mathrm{Zu}$ Vergleichszwecken haben Spezialisten weltweit Referenzsammlungen der beschriebenen Gattungen, Arten oder Varietäten angelegt. Eine der größten ist die Privatsammlung von Friedrich Hustedt, die 1968 dem Institut für Meeresforschung in Bremerhaven übergeben wurde und heute am AlfredWegener-Institut angesiedelt ist. Sie umfaßt mehr als $60000 \mathrm{mi}-$ kroskopische Präparate, 20000 Materialproben und über 4000 Literaturtitel.
B

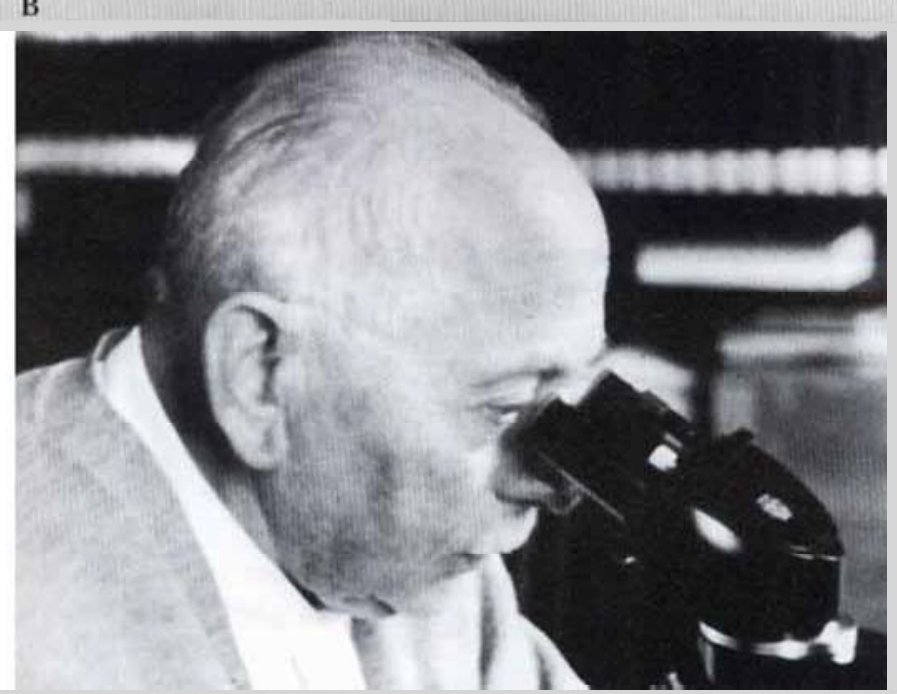

Abb. B. Dr. h. c. Friedrich Hustedt (1886 - 1968), seit 1924 Rektor einer Volksschule in Bremen, beschäftigte sich in seiner Freizeit vor allem mit Süßwasserdiatomeen, die er aus den Flüssen und Mündungsgebieten in ganz Norddeutschland mitbrachte. Hustedt wurde 1939 vom Schuldienst beurlaubt, um sich vollständig der Diatomeenforschung widmen zu können. 1963 verkaufte er seine Sammlung an das Land Bremen mit der Auflage, diese nach seinem Tode interessierten Wissenschaftlern zugänglich zu machen. Ein entsprechender Arbeitsplatz wurde 1965 am Institut für Meeresforschung in Bremerhaven eingerichtet. Zur Zeit wird an einer Datenbank gearbeitet, die auch die umfangreichen Archivinformationen enthält. Sie soll in naher Zukunft auch über das World Wide Web zugänglich sein. Fotos: R. Crawford, AlfredWegener-Institut. 
gehend unbekannt, man unterschied lediglich zwischen "amorphen und krystallinischen" Kieselsäuren. Zugleich war die systematische Erforschung der filigranen Strukturen der Diatomeenschalen seit dem Beginn des 19. Jahrhunderts bereits weit vorangeschritten. Diatomeen waren zu jener Zeit beliebte $\mathrm{Ob}$ jekte zum Testen der optischen Auflösung von Mikroskopen. Protessor Max Schultze war einer der ersten Naturforscher, der auf morphologische Ähnlichkeit der Struktur von Diatomeenschalen ,verglichen mit gewissen aus Fluorkiesel künstlich darstellbaren Kieselhäuten "verwies (Abbildung 8 und 9). Die „Kieselhäute“ wurden durch Zersetzung von gasförmigen Siliciumtetrafluorid an feuchten Oberflächen erhalten [16].

Offensichtlich war Schultze von der Idec fasziniert, die Bildung der verkieselten Diatomeenschalen auf einfache Prinzipien zurückzuführen. Er schrieb: "Jedenfalls liegt es nabe, da die erwäbnte Zeichnung [der künstlich dargestellten Kieselsäuren, Anm. d. Verf.] vielen verschiedenen Diatomeenarten in wesentlich gleicher Weise zukommt, den letzten Grund derselben vielleicht weniger in einen organischen Bildungsprocess, vielmehr in die die Abscheidung der Kieselerde ... überhaupt beherrschenden Gesetze $z u$ verlegen. Ind wern sich Krystallisation als letzter Grund nachweisen liesse, so wäre das Rätbsel gelöst".

Ein Problem, dem Schultze bei seinen Experimenten gegenüberstand, war die Tatsache, daß sich hexagonale Kugelpackungen von hexagonalen Waben der transparenten Objekte im Lichtmikroskop oftmals nicht unterscheiden lassen.

\section{Formprägung von Kieselgelen durch organische Template}

Tatsächlich gelang die gezielte Darstellung von $\mathrm{SiO}_{2}$-Materialien mit regelmäßigen $\mathrm{IO}^{\prime}$ ren erst ca. 100 Jahre später mit der Entwicklung der Zeolithe. Die technologische und wirtschaftliche Bedeutung von porösen silikatischen Materialien kann an dieser Stelle nur andeutungsweise dargestellt werden. Gegen Ende der 60er Jahre wurden die damals üblichen, amorphen Silika-Alumina-Crackkatalysatoren durch die Zcolithe verdrängt. Diesc kristallinen Alumosilikate haben seither einen Siegeszug als heterogene Katalysatoren angetreten. Zcolithe weisen formstabile periodische Anordnungen von Hohlräumen auf, die über Kanälc mitcinander verbunden sind und in denen sich Lösungsmittclmo-
7
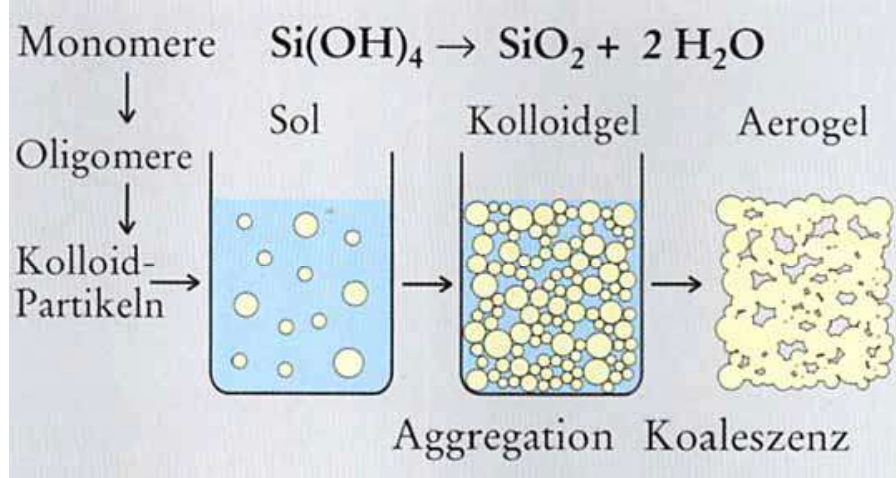

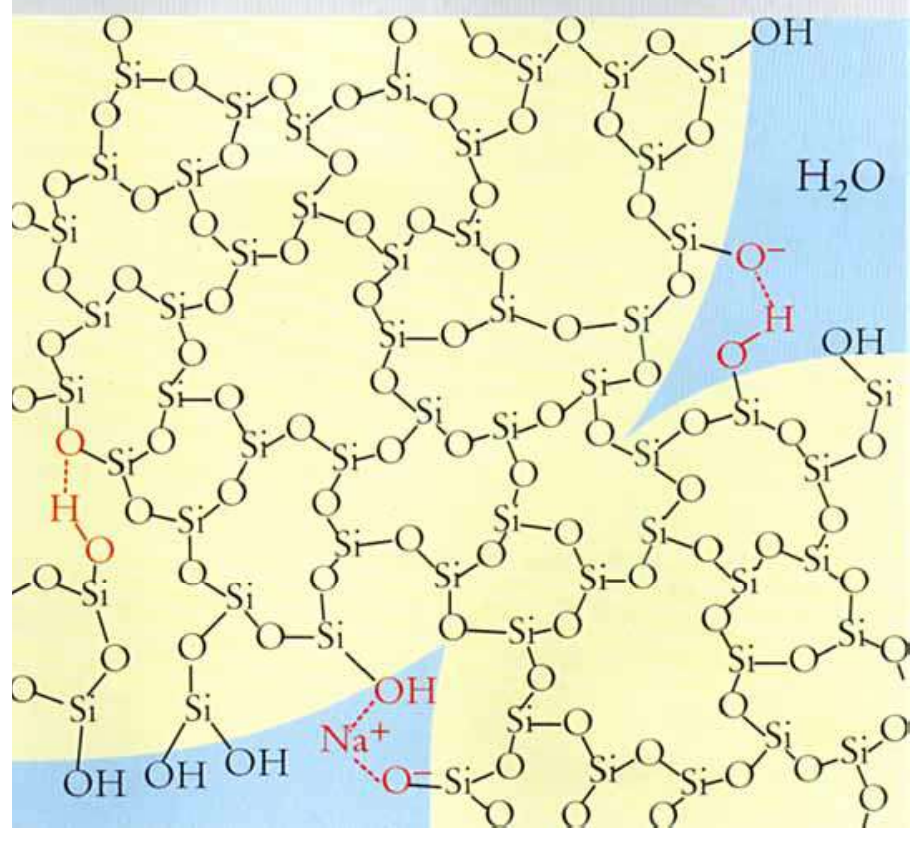

Abb. 7. Die Sol-GelChemie der Kieselsäure in wilßrigen Lösungen. Oben: Die Konde asation der monomeren Kieselsäure füh't zur Bildung vor Kolloidgelen, die aus einem Netzwe:k verknüpfter kugelförmiger Siliciumdioxidpartikeln bestehen. Dabei bildet sich zunächst ein Sol aus diskreten, gelösten Kolloidpartikeln (typische Durchmesser: 4-100 nm), die irr weiteren Verlauf unter Ausbildung kovalenter $\mathrm{Si}-\mathrm{O}$-Bindungen miteinander versch nelzen (Koaleszenz). Nach dem Entfernen des Lösungsmittels bleiben formstabile porös: Glaskörper mit hohen spezifischen Oberflächen zurück (typische Werte: $500-1000 \mathrm{~m}^{2} / \mathrm{g}$ ). Die Bedingungen, unter denen die feuchten Gele getrocknet werden, prägen die Besc affenheit der Hohlraumstrukturen: Bei den Aerogelen wird das wäßrige Lösungsmittel besonders schonend durch ein inertes Lösungsmittel verdrängt (z. B. flüssiges $\mathrm{CO}_{2}$, ,supırkritische Trocknung“), und das Kolloidskelett bleibt vollständig erhalten. Xerogele werclen dagegen durch gewöhnliche Verdampfung des Lösungsmittels erhalten, wob:i mit steigender Temperatur das Gelnetzwerk stark zunehmend sintert und die Porosi Materials abnimmt. Unten: Einzelne Gelpartikeln bestehen aus amorphem Siliciur dioxid mit folgender innerer Struktur: Jedes Si-Atom ist tetraedrisch von vier O-Atomen umgeben (das nicht abgebildete vierte O-Atom befindet sich bei der gewählten Darstellung entweder direkt ober- oder unterhalb der Si-Atome), jedes $\mathrm{O}$-Atom ist an maximal $\mathrm{z}$ wei SiAtome gebunden. Die Tetraeder aus $\mathrm{O}$-Atomen sind stets über ihre Ecken verknüf1, nicht über die Kanten oder Flächen. Durch unvollständige Kondensation bindet ein gøringer Teil der inneren Si-Atome auch Hydroxylgruppen. Die Si-Atome der Peripherie inden dagegen stets an ein oder zwei Hydroxylgruppen, die Oberfläche der Kieselgelteilc zen ist daher stark hydratisiert. Bei $\mathrm{pH}$-Werten $>3$ werden die terminalen Silanolgruppen 5 unehmend deprotoniert, und die Oberfläche der Kieselgelteilchen hat eine negative $\mathrm{N}$ sttoladung. Gelbildung erfolgt zunächst durch Aggregation der diskreten Kolloidpa tikeln über Salz-oder Wasserstoffbrücken. An der Kontaktgrenze werden die Kolloidpa tikeln dann durch fortschreitende Kondensation kovalent verknüpft [15]. 
8

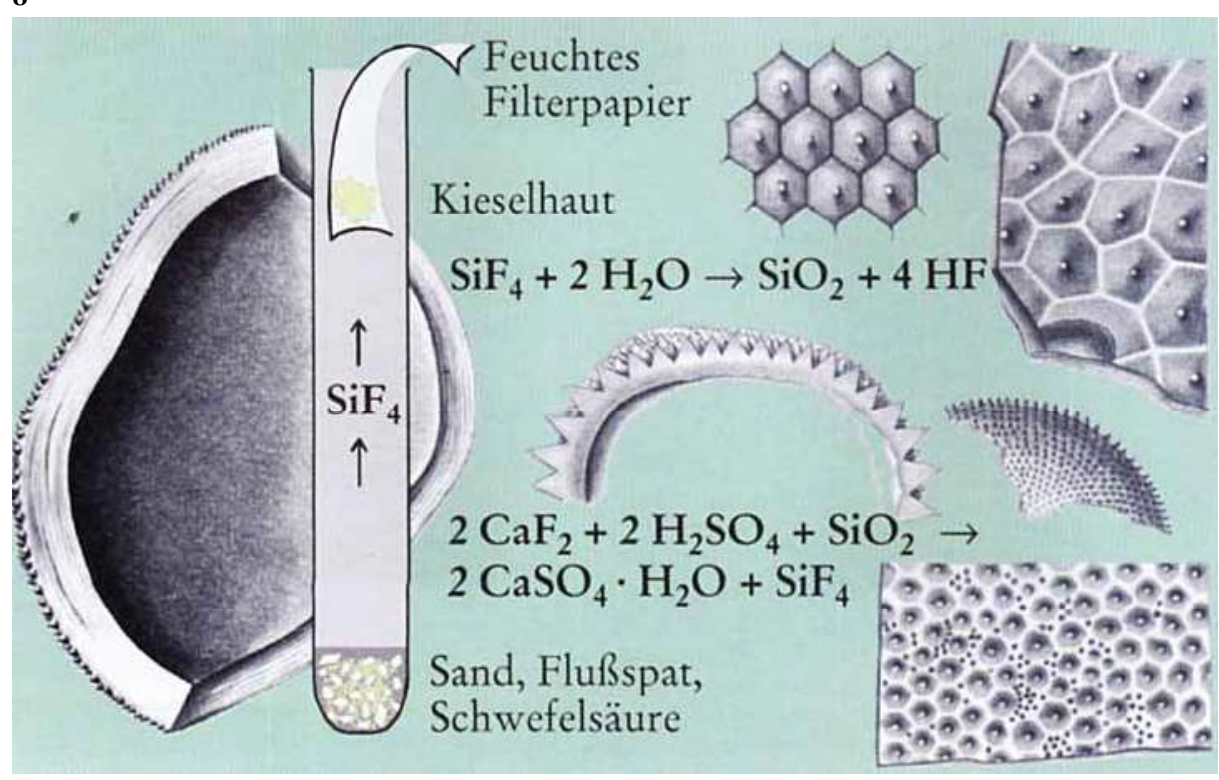

leküle frei bewegen können. Für die Synthese werden aus wäßrigen Lösungen von Silikaten und Aluminaten entstandene Gele einige Tage unter Hydrothermalbedingungen

(T $>100^{\circ} \mathrm{C}$, Druck) gealtcrt. Es zeigte sich, daß Größe und Form der Hohlraumstrukturen durch Zusatz von Templatmolekülen (z. B. quartäre Ammoniumionen) während

Tabelle 4. Formprägung von Siliciumdioxidgelen mit organischen Templaten.

$\begin{array}{lll}\text { Bezeichnung Templat, Prägematrix } \quad \text { Ordnungsparameter } & \begin{array}{l}\text { Repräsentative } \\ \text { Beispicle }\end{array}\end{array}$

Kieselgel (-)

Porosile

kleine Einzelmoleküle

Wachstumskinetik u. Vernetzung der Kolloidpartikeln

Aerogele, Xerogele [23]

kristallin, einheitlich mikroporös

(Porendurchmesser $\mathrm{d}<2 \mathrm{~nm}$ )

Mcsoporöse Makromoleküle, Kieselgele lyotrope Mesophasen, einheitlich oder multimodal mesoporös flüssigkristalline Phasen $\quad(d=2-50 \mathrm{~nm})$ Mikroemulsionen makroskopisch homogen
Makroporöse Emulsionen Kiesclgele Latexdispersionen

makroskopisch inhomogen mit perfekt hexagonalen Wabenstrukturen und einstell baren Porengrößsen $(\mathrm{d}=50 \mathrm{~nm}-10 \mu \mathrm{m})$

Diskrete Öl/Wasser-Emulsionen, meso- und makroporös $\mathrm{SiO}_{2}$-Partikeln Vesikeln (d > $50 \mathrm{~nm})$ in räumlich getrennten Bereichen, hierarchisch gegliedert
Abb. 8. Schultzes historische Versuche zur Bildung künstlicher Diatomeen. Die komplizierten und ästhetisch reizvollen Muster der Diatomeenschalen haben Naturforscher schon frühzeitig angeregt, ihre Bildung auf einfache Prinzipien zurückzuführen. Schultze beschrieb bereits 1863 Versuche zur Herstellung von dünnen Kieselmembranen, die im Lichtmikroskop Wabenstrukturen erkennen ließen. Die Kieselmembranen wurden durch Zersetzung von Siliciumtetrafluorid an einem feuchten Stück Filterpapier erhalten. Je nach Dauer der Versuche wurden unterschiedliche Strukturen erhalten: dünne, hexagonal gemusterte Membranen nach einigen Tagen, wulst- und bläschenförmige Kieselbildungen nach einigen Wochen. Die im Hintergrund abgebildeten Skizzen stammen aus der Publikation von M. Schultze [16].

der Gelierungsphase weitgehend definiert werden können. Auf diese Weise konnten formselektive Katalysatoren mit Porengrößen im Bereich von $6-13 \AA(0,6-1,3$ nm) synthetisiert werden [18].

Mit einer Porengröße von 1,3 nm scheint die Entwicklung von Zeolithen eine natürliche Grenze zu erreichen, die mit der Stabilität bestimmter Übercinheitcn zusammenhängt, welche in den Netzwerken aus spitzenverknüpften Tetraedern regelmäßig gefunden werden. Dicsc magische Grenze wurde in jüngster Zeit durch dic Entdcckung der M41Silikate durch eine industrielle Forschungsgruppe aufgehoben (Mobil Oil Corp., 1992) [20]. Diese mesoporösen, nichtkristallinen Silikate enthalten hexagonal dichtgepackte Röhrensysteme, die entstehen, wenn die Kondensationsreaktionen in den Zwischenräumen kolumnar angeordneter, zylindefförmiger Mizellen ablaufen (Abbildung 10). Durch Variation der Kettenlängen der Amphiphilalkylgruppen erhalten die geprägten Gele Poren mit gleichförmigen Durchmessern im Bereich von $2-10 \mathrm{~nm}$, deren perfekte hexagonale Anordnung sich oftmals über Bereiche von einigen Mikrometern erstreckt.

Wir erkennen hier eine deutliche Parallele zur Bildung der Muster in den Schalen der Diatomeen: Dem amorphen anorganischen Material wird durch geeignete organische Template eine Struktur aufgeprägt, die es unter Gleichgewichtsbedingungen in rein wäßriger $L_{0}$ sung nicht bildet (Tabelle 4). Voraussetzung 
für diese Art der Formprägung ist, daß die Grenzflächen zwischen der hydrophilen anorganischen und der hydrophoben organischen Phase durch "Kunstgriffe" kompatibel werden, da sonst vollständige Entmischung eintreten würde. Die organische Prägephase besteht daher vollständig oder zumindest zu einem gewisser Teil aus Amphiphilen (grenzflächenaktive Tenside, „Seifenmoleküle“), die sich in der Grenzschicht zwischen den nicht kompatiblen Phasen anreichern (vgl. Abbildung 11). Seit langem ist bekannt, daß sich geordnete Überstrukturen in Wasser-TensidGemischen ("lyotrope Mesophasen“) oder in komplizierteren Wasser/Öl/Tensid/Cotensid-Gemischen (Mikroemulsionen, „bikontinuierliche Phasen") in Abhängigkeit von der Temperatur und der Zusammensetzung ausbilden [28]. Offensichtlich entstehen solche Überstrukturen auch dann, wenn dic wäßrige Phase sukzessive durch eine anorganische Komponente ersetzt wird. Für diesen Zweck scheinen alle anorganischen Materialien geeignet zu sein, die amorphe Gel- oder Glasstrukturen bilden können. Hydratisiertes Siliciumdioxid verhält sich in dieser Hinsicht mustergültig, da sich die Oberfläche der kondensierenden Kieselgele wegen der starken Hydratisierung (adsorbierte Wassermoleküle) und der Vielzahl an terminalen Silanolgruppen sehr wasserähnlich verhält.

Bei den bisher besprochenen Kicselgelen handelt es sich durchweg um Materialen, die bei einer gegebenen Zusammensetzung eine einheitliche innere Struktur ausbilden und deren äußere Begrenzung durch die Abmessungen des Reaktionsgrefäßes limitiert wird. Eine morphologische Ähnlichkeit zu den Schalenstrukturen der Diatomeen ist zunächst nicht feststellbar. Einen Schritt in Richtung auf die biomimetische Synthese gegliederter und diskreter Schalenstrukturen gelang verschiedenen Forschergruppen in jünster Zeit $[27,29]$, wobei hier die Strategie von Schüth und Mitarbeitern kurz vorgestellt werden soll [30]. Diese erhielten KieselgelHohlkugeln mit Durchmessern von $1-100$ $\mu \mathrm{m}$ durch Hydrolyse eines Siliciumprecursors in der Öl/Wasser-Grenzschicht einer wäßrigen Makroemulsion (Abbildung 13). Von besonderem Interesse ist bei diesem Verfahren, daß die auf das Öltröpfehen aufwachsende Kieselgelschicht zusätzlich mesoskopisch strukturiert ist. Offensichtlich hat der Zusatz des Amphiphils hier zwei Funktionen: Dic Stabilisierung der Öltröpfchenemulsion auf der makroskopischen Ebenc und dic Bildung ciner lyotropen Mesophase mit dem

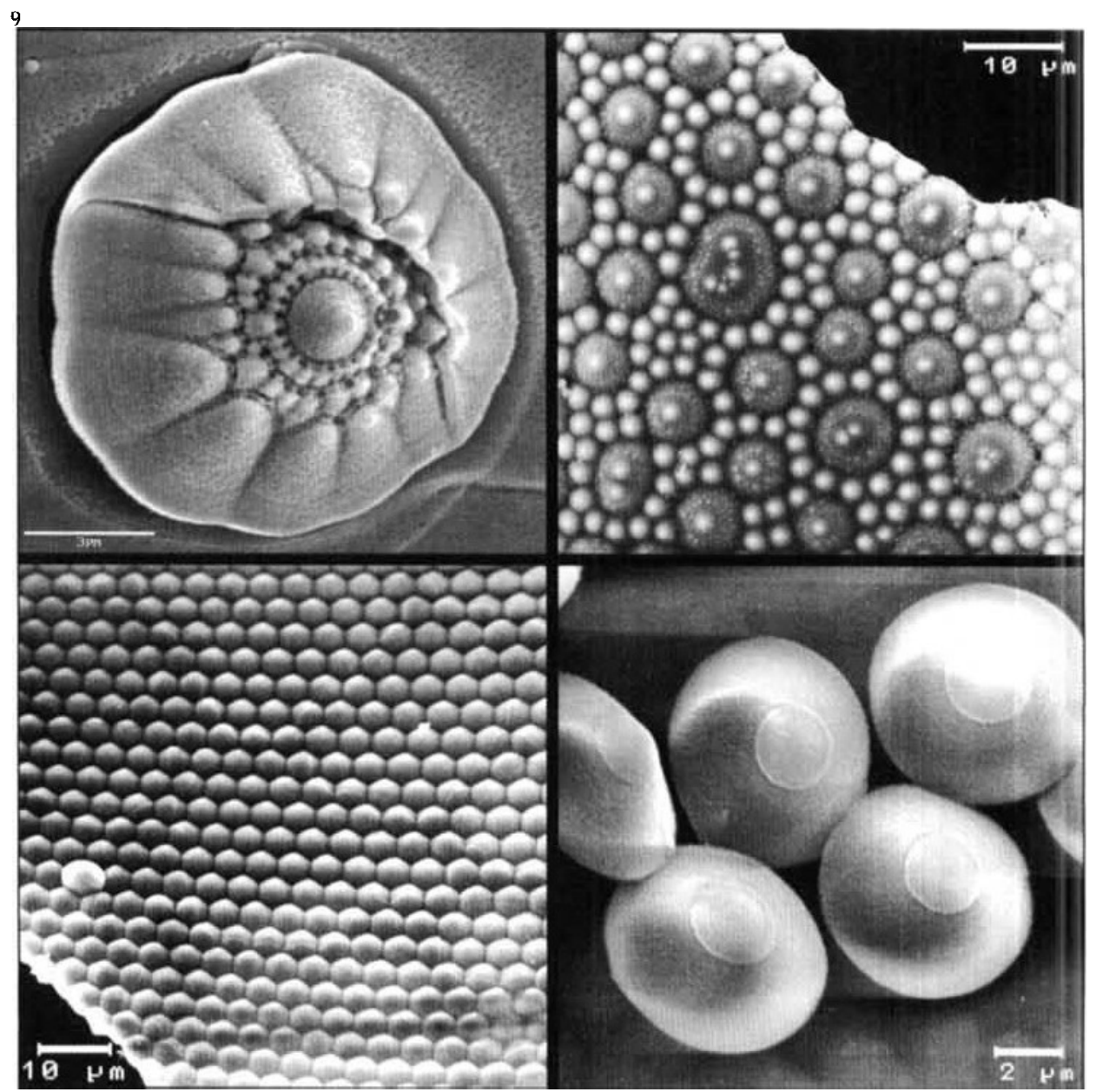

10
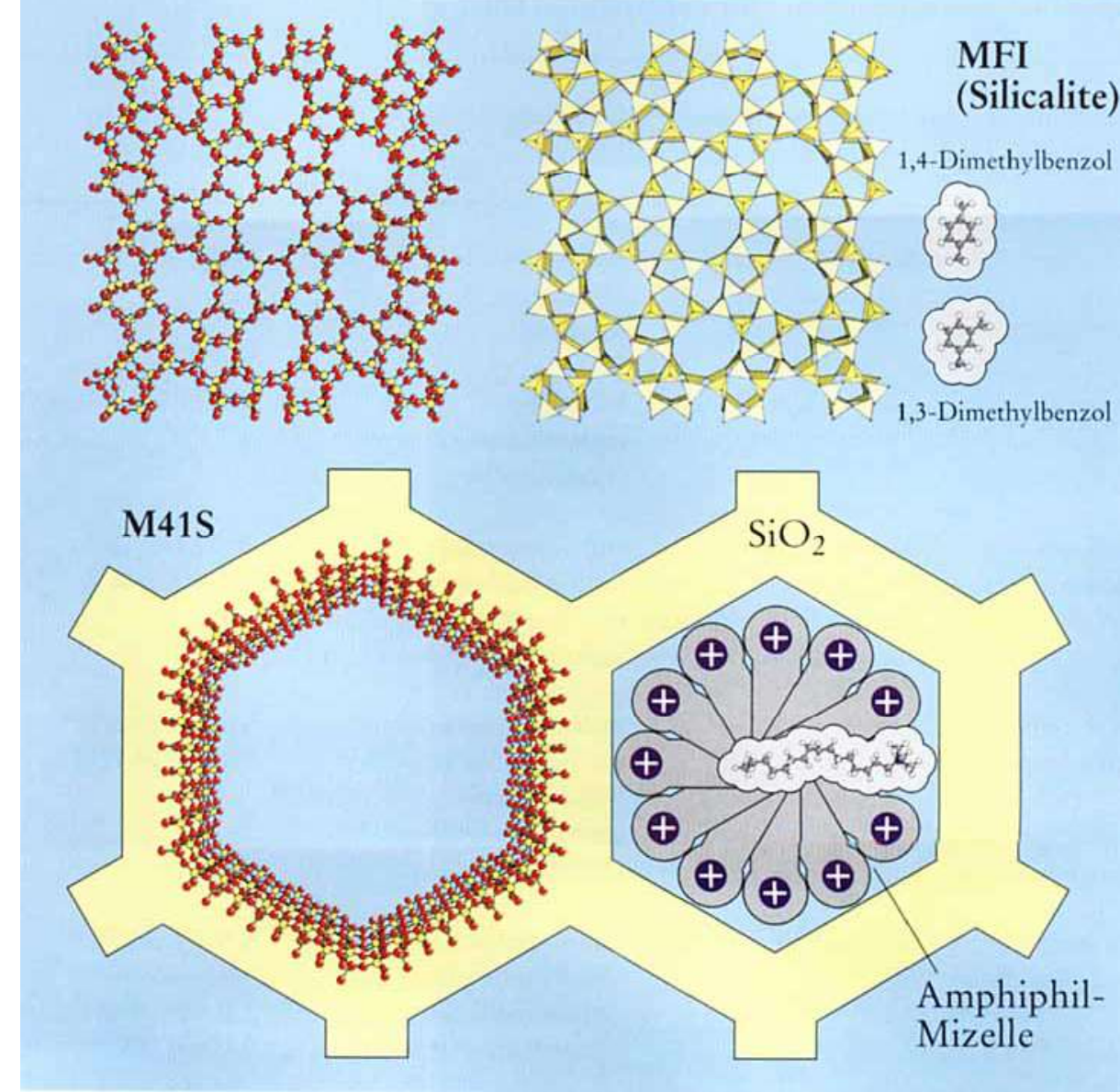
Abb. 9. Rasterelektronenmikroskopische Aufnahmen von Kieselhäuten, die nach dem Verfahren von Schultze hergestellt wurden. Wabenmuster entstehen durch dichte Schichtpackungen kolloidaler Siliciumdioxidpartikeln. Vereinzelt werden auch Aggregate mit komplizierterer Morphologie beobachtet, z. B. "Seepocken“" (links oben) oder „Kegelschnecken “ (rechts unten). Hexagonal perforierte Schichten mit einer Ähnlichkeit zu den gekammerten Diatomeenvalven wurden bislang nicht gefunden [17].

Abb. 10. Poröse, künstlich erzeugte Siliciumdioxidfestkörper im Strukturvergleich. Oben: Kugel-Stab- und Polyederdarstellung der Kristallstruktur von Silicalite (Blick in die 10-gliedrigen Kanäle entlang der kristallographischen $y$-Achse, mit einem Porendurchmesser von ca. 5,5 A) [19]. Vom Strukturtyp MFI leiten sich eine Reihe mikroporöser Zeolithe ab, die bei petrochemischen Anwendungen eine wichtige Rolle spielen. Ersetzt man z. B. in der Struktur von Silicalite einen Teil der $\mathrm{Si}^{4+}$. durch $\mathrm{Al}^{3+}$-Kationen, so erhält man ZSM-5 $\left(\mathrm{Na}_{\mathrm{n}}\left[\mathrm{Al}_{\mathrm{n}} \mathrm{Si}_{96-\mathrm{n}} \mathrm{O}_{192}\right] \times 16 \mathrm{H}_{2} \mathrm{O} ; \mathrm{n}<27\right)$. Dieser formselektive Katalysator wird $\mathbf{u}$. a. zur Isomerisierung von Xylol eingesetzt, wobei der Anteil des gewünschten paraIsomers im Gemisch gesteigert wird. Unten: Ausschnitt aus der idealisierten Struktur eines mesoporösen M41-Silikates. Diese Siliciumdioxidfestkörper entstehen bei der templatgesteuerten Kondensation von Kieselsäure in wäßrigen Mikroemulsionen. Amphiphile wie das hier abgebildete Hexadecyl-trimethylammoniumkation bilden bei bestimmten Konzentrationen langgestreckte zylinderförmige Mizellen, die sich in hexagonal dicht gepackten Reihen anordnen können. Die Kondensation der Kieselsäure findet dann in den Zwischenräumen der gepackten Mizellen statt. Nach dem Entfernen des organischen Templates wird ein amorphes Siliciumdioxid erhalten, das offene, periodisch angeordnete Kanäle mit Porendurchmessern von $40 \AA$ enthält. Systematische Untersuchungen zeigen, daß die Porenweite durch Variation des Amphiphils und durch Zusatz organischer Hilfsstoffe beliebig zwischen 15 und $100 \AA$ eingestellt wcrden kann [20].
11

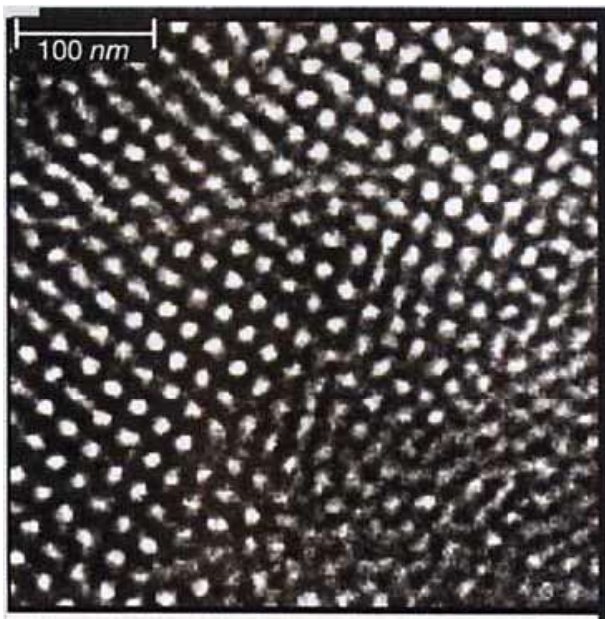

a)

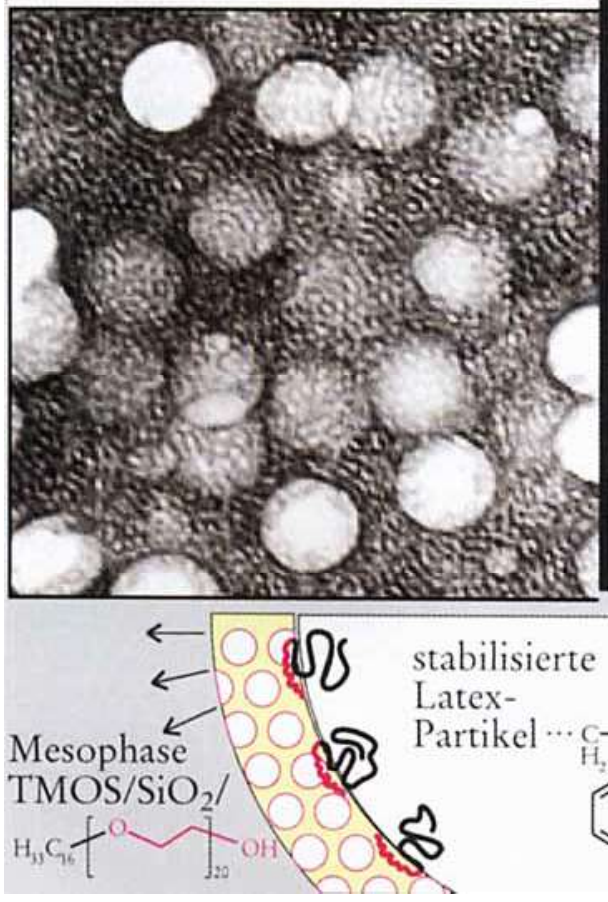

b) Phase
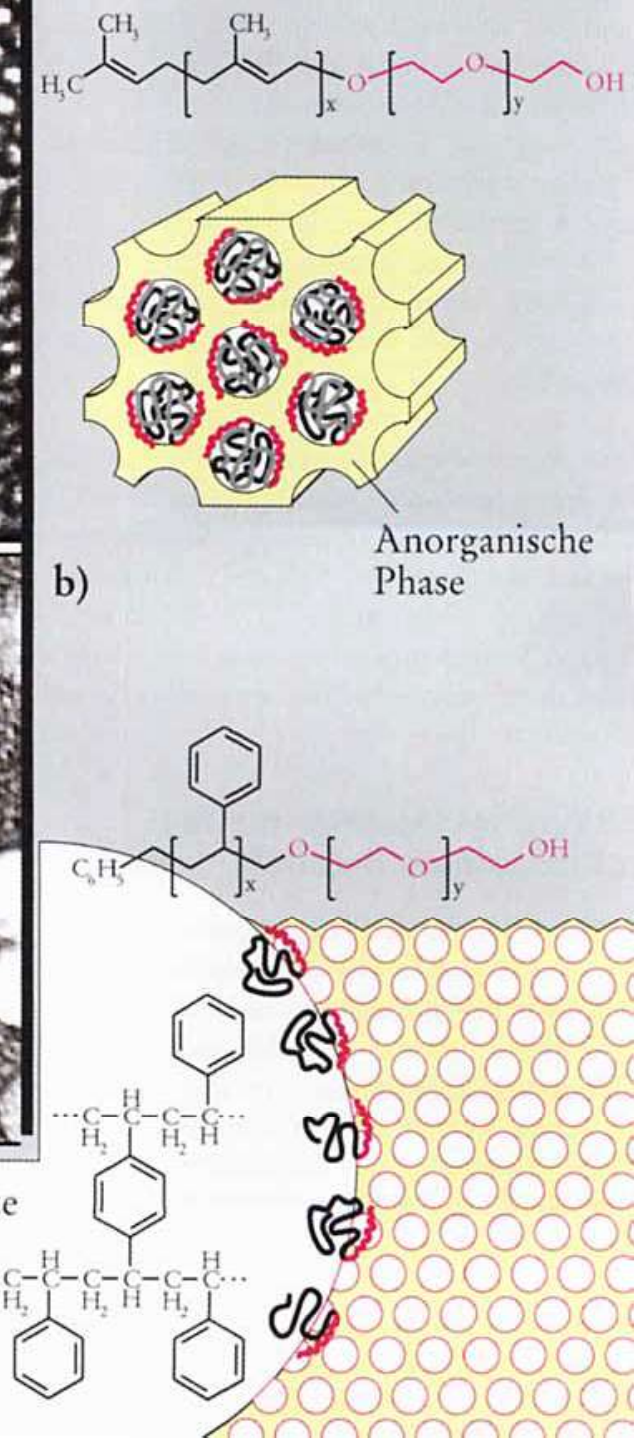

Abb. 11. Seit der Präsentation der M41-Silikate boomt die Entwicklung mesoporöser Materialien, wobei zunehmend verfeinerte Template zum Einsatz kommen und die Untersuchungen auch auf andere anorganische Materialien ausgedehnt werden. Zwei aktuelle Beispiele geben einen Ausblick auf zukünftige Aspekte der templatgesteuerten Festkörpersynthesen:

a) Prägung von anorganischen Festkörpermatrices durch supramolekular organisierte AB-Blockcopolymere [21],

b) gleichzeitige Prägung von Festkörpern durch organische Template auf unterschiedlichen Größenebenen [22]. Zum Einsatz kommen Latexteilchen, deren Oberfläche durch Verankerung nichtionischer Tenside hydrophil modifiziert wurde, die in ein hierzu kompatibles, lyotrop organisiertes System aus Amphiphil und dem kondensierenden Kieselgel gebracht werden. Nach dem Entfernen der organischen Bestandteile verbleibt ein schwammförmiges Siliciumdioxid mit einer bimodalen Verteilung der Porengrößen.

Vom Prinzip her ähnliche selbstorganisierte Vorgänge spielen sich vermutlich auch bei der Bildung der hierarchisch gegliederten Schalen der Diatomeen ab, wobei Biomembranen und Vesikeln die Rolle der organischen Template übernehmen. (TEM-Aufnahmen: A. Du Chesne, U. Wiesner, MPI für Polymerforschung, Mainz; und C. Goeltner, MPI für Kolloid- und Grenzflächenforschung, Berlin). 
kondensierenden Kieselgel in einer räumlich begrenzten Schicht. Es findet also ein Prozeß statt, der dem anorganischen Material simultan eine äußere Form und eine innere Struktur aufprägt. Wie die Autoren ferner berichten, hängt die Morphologie der erhaltenen Teilchen stark von den Synthesebedingungen ab. So werden bei niedrigen Rührgeschwindigkeiten faserige Strukturen erhalten, die bei Erhöhung der Rührgeschwindigkeit zunehmend in Partikel mit kugelförmiger Gestalt übcrgchen.

Wie in Abbildung 12 schematisch dargestellt ist, liegt hier bereits cin funktionales Modellsystem vor, das verschiedene Aspekte der Schalenmorphogenese bei den Diatomeen imitiers: cin - wenn auch einfacher - Siliciummetabolismus, eine selbstorganisierte Formentstehung und ein selbstterminiertes Schalenwachstum.

\section{Zukünftige Aspekte der bio- mimetischen Materialforschung}

Anhand der Schalenmorphogenese bei den Diatomeen wurde hier exemplarisch dargestellt, wie Erkenntnisse aus den biologischen Disziplinen und den Materialwissenschaften ineinandergreifen können und sich innovative Strategien zum Aufbau ncuartiger gegliederter Materialien aus Siliciumdioxid ergeben. Nach den Prinzipien der Biomineralisation lassen sich auch Systeme aus Matcrialien aufbaucn, die in der Natur nicht vorkommen. Deutliche Erfolge wurden hier in jüngster Zeit auf dem Gebiet der gezielten Mineralisation extrem cinheitlicher Metallcluster und Kristallite in biomimetischen Wirtsmatrizen [32] und bei der Synthese mesoporöser Metalloxide erreicht [33].

Zwar sind wir noch weit entfernt von der routinemäßigen Herstellung anorganischer Materialicn, dic cine den natürlichen Systemen vergleichbare Gliederung aufweisen ( $\mathrm{vgl}$. Abbildung 1), doch lohnt es sich, über biomimetische Verfahren zur Erzeugung komplex strukturierter Materialien nachzudenken [34].

Dieser logische Weg - die Analyse der (molekular-)biologischen Aspekte, die Abstraktion der biologischen Vorgänge zu einem vereinfachten Modell und dic Umsctzung der Modellvorstcllungen in ein konkretes Syntheseprinzip - bildet das Paradigma für den neuen, sich rasch etablierenden Forschungszweig der biomimetischen Materialwissenschaften [35].

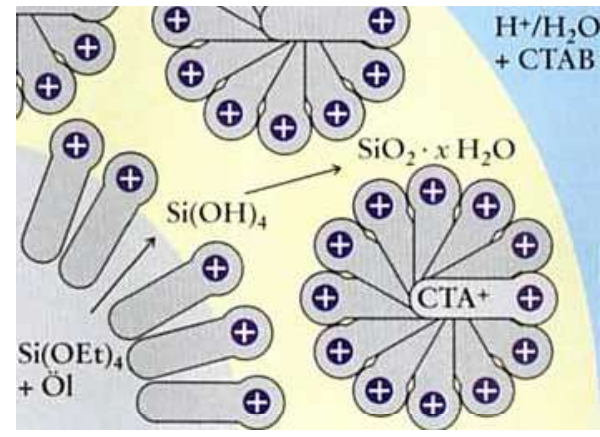

Abb. 12. Primitive Schalenstrukturen können entstehen, wenn die Ausbildung der anorganischen Phase auf einen schmalen räumlichen Bereich begrenzt wird und die Ausgangsstoffe für das weitere Wachstum der Mineralphase limitiert sind. Bei der katalysierten Hydrolyse von Tetraethoxysilan $\left(\mathrm{Si}(\mathrm{OEt})_{4}\right)$ in einer Öl/Wasser-Makroemulsion erhielten Schüth und Mitarbeiter poröse Hohlkugeln aus Kieselgel [30]. Die Entstehung der Kugeln konnte lichtoptisch verfolgt werden. Demnach diffundiert der in dem Öltröpfchen vorhandene Kieselsäureprecursor an die Öl/Wasser-Grenzfläche, wo eine rasche Hydrolyse stattfindet. Bei der weiteren Kondensation der Kieselsäure wächst das Kieselgel als dünne Schicht um das Öltröpfchen. Die Feinstruktur der Kieselhaut wird vermutlich durch die Anwesenheit der Amphiphilmoleküle (CTAB Cetyltrimethyl-ammoniumbromid) verursacht: Diese stabilisieren nicht nur das Öltröpfchen in dem wäßrigen Reaktionsmedium, sondern bilden mit dem kondensierenden Kieselgel eine geordnete Mesophase.

Unser Verständnis über die molekularen Grundlagen der Biomineralisation, inbesondere über die dynamischen Vorgänge der $\mathrm{Mu}$ sterbildung und der Formentstehung, ist zur Zeit noch rudimentär, Es ist aber abzusehen, daß sich die chemische Grundlagenforschung in zunehmendem Maße dem Phänomen der Selbstorganisation und den chemischen Prinzipien der Morphogenese zuwenden wird.

\section{Danksagung}

Mein Dank gilt allen Personen, die mir Bilder für den vorliegenden Aufsatz zur Verfügung gestellt haben. Mein besondcrer Dank gilt Frau Hannelore Halliger für die freundliche Hilfe und Beratung bei der Anzucht von Diatomecnkulturen. Dem Fonds der Chemischen Industrie (FCI) danke ich für dic finanziclle Unterstützung meiner Arbeit durch ein Liebig-Stipendium.

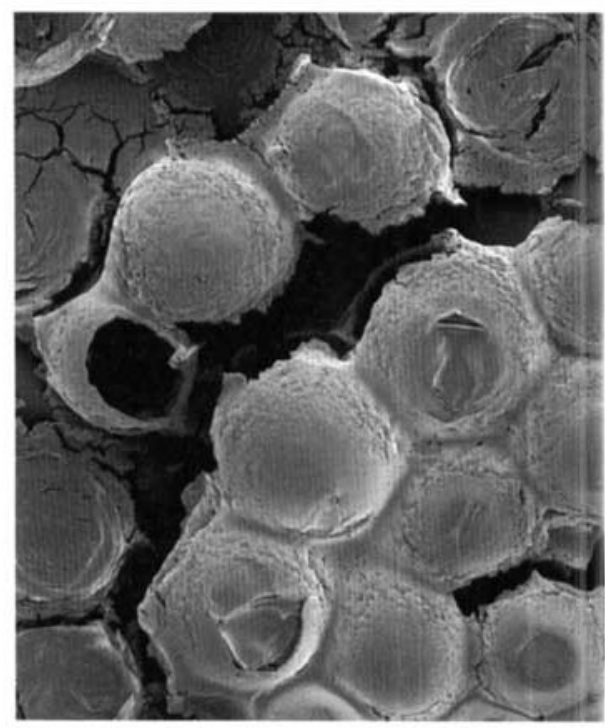

Abb. 13. Poröse Kugeln aus Siliciunıdioxid, die nach dem Verfahren von Schi th und Mitarbeitern gewonnen wurden [30, 31]. (REM, Durchmesser der Kugeln $z$ vischen 60 und $100 \mu \mathrm{m}$ ).

\section{Summary}

Diatoms are unicellular organisms which possess a unique silicified cell wall. ' $T$ ' $x$ cir intricate structures have inspired the ir agination of naturalists and scientists throughout centuries. However, molecular biolog cal details about silica metabolic pathways itill are obscure. To find out how soft matter $c$ mn selforganize and create finite solid shafes and complex patterns is certainly one of the most challenging scientific problems today. ispccially chemists just have started to unrovel the mysteries of chemical morphogenesis.

The article describes how cellular events occuring at different steps of diatom cell wall formation and silica biomineralization may serve scicntists as a model to create inn vative biomimetic strategies for the synth sis of hicrarchically structured materials. 


\section{Literatur}

[1] Einfach ausgedrückt handelt es sich dabei um die Erforschung des gesamtheitlichen Verhaltens eines Systems, das sich aus einer endlichen Anzahl diskreter, mitcinander wechselwirkender Komponenten zusammensetzt. Die geeignete Kombination kann zu cinem komplexen Verhalten des Gesamtsystems führen, das aus der Kenntnis der Eigenschaften der einzelnen Komponenten allein nicht vorhersagbar ist. Den theoretischen Überbau hierzu liefert die Chaos-oder auch Komplexitätstheorie. Sehr schöne allgemeine und verständliche Einführungen in diverse Bereiche der Selbstorganisationsforschung findet man z. B. in: Muster des Lebendigen, (Hrsg.: A. Deutsch), Vieweg, Braunschweig, 1994, und auch: Der Öltropfen im Wasser. Chaos, Komplexität, Selbstorganisation in Natur und Gesellschaft, S. Kauffman, Piper, München, 1996. Über dic wissenschaftstheoretischen Hintergründe informiert z. B.: F. Mußmann, Komplexe Natur Komplexe Wissenschaft, Leske + Budrich, Opladen, 1995.

[2] S. Mann, Cbem. unserer Zeii 1986, 20,69.

[3] N. Kröger, C. Bergsdorf und M. Sumper, Eur.J. Biochem. 1996, 239, 259.

[4] R. E. Hecky, K. Mopper, P. Kilham und E.T. Degens, Mar. Biol. 1973, 19, 323.

[5] M. E. Marsh, Protoplasma 1994, 177, 108.

[6] L. Addadi und S. Weiner, Angew. Chem. $1992,104,159$.

[7] A. George, L. Bannon, B. Sabsay, J. W. Dillon, J. Malone, $\Lambda$. Veis, N. A. Jenkins, D. J. Gilbert und N. G. Copcland, J. Biol. Chem. 1996, 271, 32869 .

[8] Silicon and Siliceous Structures in Biologal Systems, (Hrsg.: T. L. Simpson, B. E. Volcani), 1. Aufl., Springer, New York, 1981.

[9] Silica, Ullmann's Encyclopedia of Industrial Chemistry, Vol. A 23, 583, VCH, 1993.

[10] A.-M. M. Schmid, Protoplasma 1994, $181,43$.

[11] a) Algen, 3. Aufl., (Irsg.: C. van den Hoek, H. M. Jahns und D. G. Mann), S. 123, Thieme, Stuttgart, 1993. b) H. A. von Stosch und G. Drebes, Helgol. Wiss. Mecresunters. 1964, 11, 209.

[12] M. Hildebrand, B. F. Volcani, W. Gassmann und J. I. Schroeder, Nature 1997, 385, 688.

[13] C. W. Sullivan, Silicification by diatoms in Silicon biochemistry, Ciba Foundation Symposium 121, 59, Wilcy, Chichester, 1986

[14] R. Gordon und R. W. Drum, Int. Rev. Cytology 1994, 150, 243.

[15] R. K. Iler, The Chemistry of Silica, Wiley, New York, 1979.

[16] M. Schultze, Verbandl. des naturbist. Vereins der preuss. Rheinlande u. Westphalens, 1863, $X X$, Neue Folge $X, 1$.

[17] D. Volkmer, unveröffentlichte Ergebnisse.

[18] A. Corma und A. Martinez, Adv. Maler. $1995,7,137$.

[19] E. M. Flanigen, J. M. Bennett, R. W. Grose, J. P. Cohen, R. L. Patton, R. M. Kirchner und J. V. Smith, Nature 1978, 271, 512.

[20] C. T. Kresge, M. E. Leonowic W. J. Roth, J. C. Vartuli und J. S. Beck, Nature $1992,359,710$.

[21] M. Templin, A. Franck, A. Du Chesne, H. Leist, Y. Zhang, R. Ulrich, V. Schädler und U. Wiesner, Science 1997, 278, 1795.

[22] M. Antonietti, B. Berton, C. Göltner und H.-P. Hentze, Ado. Mater. 1998, 10, 154.

[23] C. J. Brinker und G. W. Scherer, Sol-Gel Science, Kap. 9, S. 515 ff., Academic Press, London, 1990.

[24] H. Gerke und H. Gies, Z. Kristallogr. $1984,166,11$.

[25] A. Imhof, D. J. Pine, Nature 1997, 389, 948.

[26] B. T. Holland, C. F. Blanford und A. Stcin, Science 1998, 281, 538.

[27] a) H. Yang, N. Coombs und G. A. Ozin, Nature 1997, 386, 692. b) G. A. Ozin und S. Oliver, Adv. Mater. 1995, 11, 943.

[28] H. Hoffmann und W. Ulbricht, Chem. unserer Zeit 1995, 29, 76.
[29] a) P. T. Tanev und T. J. Pinnavaia, Science 1996, 271, 1276. b) S. Oliver, A. Kuperman, N. Coombs, A. Lough und G. A. Ozin, Nature 1995, 378, 47. c) R. Kniep und S. Busch, Angew. Chem. 1996, 108, 2788. c) D. Walsh und S. Mann, Nature 1995, 377, 320.

[30] S. Schacht, Q. Huo, I. G. Voigt-Martin, G. D. Stucky und F. Schüth, Science 1996, 273,768 .

[31] Q. Huo, D. Zhao, J. Feng, K. Weston, S. K. Buratto, G. D. Stucky, S. Schacht und F. Schüth, Adv. Mater. 1997, 9, 974.

[32] M. Möller, J. P. Spatz, Curr. Opin. in Col. Interface Sci. 1997, 2, 177.

[33] P. Behrens, Angew. Chem. 1996, 108 , 561.

[34] a) S. Mann und G. A. Ozin, Nature 1996, 382, 313. b) G. A. Ozin, Acc, Chem. Res. $1996,30,17$.

[35] Biomimetic Materials Chemistry, (Hrsg.: S. Mann), 1. Aufl., VCH, Weinheim, 1996.

[36] The Diatoms: Biology \& Morphology of the genera, (Hrsg.: F. E. Round, R. M Crawford und D. G. Mann) Cambridge University Press, Cambridge, 1992.

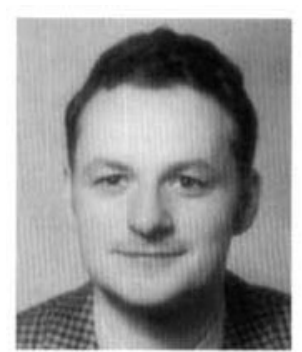

Dr. rcr. nat. Dirk Volkmer, geboren 1965 in Gladbeck, Chemiestudium an der Westfälischen Wilhelms-Universität Münster, 1994 Promotion bei Bernt Krebs, 1995/1996 Postdoctoral Fellow bei Jean-Marie Lehn in Strasbourg (Frankreich). Seit 1997 Wissenschaftlicher Assistent am Lehrstuhl Anorganische Chemie 1 der Universität Bielefeld bei Achim Müller. Zur Zeit als Gastwissenschaftler bei Lia Addadi und Stephen Weiner am Weizmann Institute of Science (Israel). Arbeitsgebiete: Grundlagen der Biomineralisation, biologisch inspirierte Materialsynthesen und enzymmimetische (bioanorganische) Chemie.

Korrespondenzadresse:

Dr. Dirk Volkmer, Universität Bielefeld, Anorganische Chemie 1, Postfach 100131, D 33501 Bielefeld, E-mail: dvolkmer@uni-bielefeld.de 